\title{
Analysis of atomic-orbital basis sets from the projection of plane-wave results
}

\author{
Daniel Sánchez-Portal, Emilio Artacho, and José M. Soler \\ Instituto de Ciencia de Materiales Nicolás Cabrera and Departamento de Fúsica de la Materia Condensada, C-III \\ Universidad Autónoma de Madrid, 28049 Madrid, Spain.
}

(October 17, 2018)

\begin{abstract}
The projection of the eigenfunctions obtained in standard plane-wave first-principle calculations is used for analyzing atomic-orbital basis sets. The "spilling" defining the error in such a projection allows the evaluation of the quality of an atomic-orbital basis set for a given system and its systematic variational optimization. The same projection allows obtaining the band structure and the Hamiltonian matrix elements in the previously optimized atomic basis. The spilling is shown to correlate with the mean square error in the energy bands, indicating that the basis optimization via spilling minimization is qualitatively equivalent to the energy-minimization scheme, but involves a much smaller computational effort. The spilling minimization also allows the optimization of bases for uses different than total-energy calculations, like the description of the band gap in semiconductors. The method is applied to the characterization of finite-range pseudo-atomic orbitals [O. F. Sankey and D. J. Niklewski, Phys. Rev. B 40, 3979 (1989)] in comparison to infinite-range pseudo-atomic and Slater-type orbitals. The bases are evaluated and optimized for several zincblende semiconductors and for aluminum. The quality of the finite-range orbitals is found to be perfectly comparable to the others with the advantage of a limited range of interacions. A simple scheme is proposed to expand the basis without increasing the range. It is found that a double- $z$ basis substantially improves the basis performance on diamond, whereas $d$-polarization is required for $\mathrm{Si}$ and $\mathrm{Al}$ for similar results. Finally, the projection allows the chemical analysis of the plane-wave results via population analysis on the previously optimized atomic basis. The spilling can partially control the intrinsic arbitraryness of the population analysis.
\end{abstract}

\section{INTRODUCTION}

One of the several approximations needed for electronic structure calculations of solids is the truncation of the one-particle Hilbert space. Choosing an appropiate basis is critical for obtaining high-quality results. There are schemes based on localized, extended, or mixed bases. Most of the methods of the first group use atomic-like bases, the eigenfunctions being obtained as lipear combinations of atomic orbitals (LCAO methods). 1 The most widely used among the extended is the plane-wave basis.

Plane waves (PW) together with pseudopotentials have shown to offer a very successful calculation scheme for a very large number of applications. Particulary since the development of $a b$ initio pseudopotentials $\mathrm{PW}$ have provided the tool forvery accurate calculation of different properties of solids. 3 PW are quite simple to implement and the convergence of the calculations can be controlled with a single parameter, the planewave cut-off. The PW basis is also independent of atomic positions, which is very convenient for the coding. However, in addition to these advantages, plane waves have important drawbacks, namely (i) the imposed translational invariance (supercells for non-periodic systems), and (ii) their inefficiency in basis size.

LCAO methods are much more efficient in the size of the required basis. This is a very important advantage for the calculation of large systems. Moreover, they have shown to be very suitable for order-N methods 3 in which the computational effort scales linearly with the size of the system. However, this large reduction in the size of the basis is obviously accompanied by a potential loss of completeness which can affect the results. It is then necessary to choose an appropiate basis to obtain accurate results. Nevertheless, it is important to stress that, once a high-quality atomic basis set has been chosen, calculations can be performed with an accuracy perfectly comparable to that of plane waves and with a large reduction of CPU time and memory.

Several methods have been developed to optimize LCAO basis sets. Most of them are based in two procedures: ( $i$ ) minimization of total energies of atoms, molecules, or solids, 0 and (ii) minimization of differences in the energy bands comparing with the experiment or with PW calculations. 1.6 We present here a projection method that links PW and LCAO schemes and provides: $(i)$ a systematic way to characterize the quality of an atomic basis and therefore a way to optimize it, (ii) LCAO energy bands and Hamiltonian and overlap matrix elements, and (iii) chemical information through population analysis. The key of the optimization method is to measure the ability of a set of atomic orbitals to represent the actual eigenstates of the PW calculation of a system. By minimizing the error in the projection of the eigenstates the atomic basis can be optimized for that particular system. This scheme is related to methods of quantum chemistry where a relatively small LCAO basis set is optimized by minimizing the distance (maximizing the overlap) of its subspage to the one spanned by another, larger LCAO basis 8 The scheme used in 
this paper was already outlined in Ref. 9, the projection technique for bands being already sketched by Chadi in Ref. 6.

Besides the projection optimization, the possibility of calculating LCAO band structures and matrix elements allows the characterization of (i) the energetics, and (ii) the range of interactions of the basis. Particulary for this purpose non-orthogonalized atomic basis sets are more adequate, since the interaction parameters have proved to be much more transferable and to have shorter range than for orthogonal bases.10.11 The direct use of atomic orbitals in condensed systems gives non-orthogonal basis sets naturally. These non-orthogopgl atomic basis sets will be used throughout this paper.12

In the present paper, this method of analysis and optimization is applied to the characterization of basis sets made of the finte-range pseudg-atomic orbitals introduced by Sankey and Niklewski 13 They are compared with the infinite-range pseudo-atomic orbitals 14 and Slater-type orbitals. Both minimal and expanded bases are explored as applied to several zincblende semiconductors, as well as to aluminum.

The optimized basis can be used for the calculation of larger systems, where plane-wave calculations are impractical. Besides that possible use, they can also be used directly for the analysis of the PW results themselves. 9 The chemical language of population analysis is not accesible to the PW scheme. The projection into the optimum LCAO basis allows its application, to compute atomic charges, bond charges, and charge transfers. In a similar way as the decomposition of the total charge into orbital populations, the density of states can also be projected into atomic orbitals to give local densities of states. It is shown here how the optimization of the basis is important for obtaining sensible values of the charges, and also how the projection can be used to partially control the intrinsic arbitraryness of any population analysis.

The structure of this paper is as follows. A brief description of the method to characterize and optimize the atomic basis is given in section II, as well as a description of the way of performing the population analisys and the band structure calculations. Section III describes the main characteristics of the $a b$ initio PW calculations used here. A description of the different types of atomic orbitals analyzed in this paper can be found in section IV. Section V proposes the spilling as a parameter to characterize basis sets and their correlations with other quantities. Section VI contains the results of the analisys and optimization of the differents sets of atomic orbitals. Population analysis is discussed in section VII. We end with the conclusions in section VIII.

\section{DESCRIPTION OF THE METHOD}

The method presented in this paper is based on projection techniques. 6 Given the results of a PW calcula- tion, the projection of the calculated eigenstates into an atomic basis can provide information about this basis set and about the PW results. A good basis must be able to represent the essential features of the PW eigenstates. Once the PW calculation has been performed for the system of interest, the projection process is much less costly than the self-consistent calculation itself, and many different basis sets can be analyzed and optimized for one single PW calculation, a much more economic procedure than performing a full self-consistent calculation for each trial basis set.

On the base of our method we have the definition of the spilling parameter 9 Given a PW calculation we define the spilling $\mathcal{S}_{\Omega}$ for a given local basis set as

$$
\mathcal{S}_{\Omega}=\frac{1}{N_{k}} \frac{1}{N_{\alpha}} \sum_{k}^{N_{k}} \sum_{\alpha=1}^{N_{\alpha}}\left\langle\psi_{\alpha}(\mathbf{k})|(1-P(\mathbf{k}))| \psi_{\alpha}(\mathbf{k})\right\rangle,
$$

where $\left|\psi_{\alpha}(\mathbf{k})\right\rangle$ are the PW calculated eigenstates, and $N_{k}$ and $N_{\alpha}$ are the number of calculated $\mathbf{k}$ points in the Brillouin zone and the number of bands considered, respectively, and $\Omega$ is the specific subspace spanned by the eigenstates included in the sum (defined by $N_{k}$ and $\left.N_{\alpha}\right) . P(\mathbf{k})$ is the projector operator into the subspace of Bloch functions of wave vector $\mathbf{k}$, generated by the atomic basis, and defined as usual for non-orthogonal basis, 12

$$
P(\mathbf{k})=\sum_{\mu}\left|\phi_{\mu}(\mathbf{k})\right\rangle\left\langle\phi^{\mu}(\mathbf{k})\left|=\sum_{\mu \nu}\right| \phi_{\mu}(\mathbf{k})\right\rangle S_{\mu \nu}^{-1}(\mathbf{k})\left\langle\phi_{\nu}(\mathbf{k})\right|,
$$

where

$$
\left\langle\mathbf{r} \mid \phi_{\mu}(\mathbf{k})\right\rangle=\sum_{\mathbf{R}} \phi_{\mu}\left(\mathbf{r}-\mathbf{r}_{\mu}-\mathbf{R}\right) e^{i \mathbf{k}\left(\mathbf{r}_{\mu}+\mathbf{R}\right)}
$$

$\phi_{\mu}(\mathbf{r})$ being the atomic orbitals, $\mathbf{r}_{\mu}$ the atomic coordinates in the unit cell, and $\mathbf{R}$ the lattice vectors.

$$
S_{\mu \nu}(\mathbf{k})=\left\langle\phi_{\mu}(\mathbf{k}) \mid \phi_{\nu}(\mathbf{k})\right\rangle
$$

is the overlap matrix of the atomic basis. The vectors $\left|\phi^{\mu}(\mathbf{k})\right\rangle$ constitute the dual of the atomic basis, that satisfy

$$
\left\langle\phi^{\mu}(\mathbf{k}) \mid \phi_{\nu}(\mathbf{k})\right\rangle=\left\langle\phi_{\mu}(\mathbf{k}) \mid \phi^{\nu}(\mathbf{k})\right\rangle=\delta_{\mu \nu}
$$

$\mathcal{S}_{\Omega}$ is a parameter that varies between 0 and 1 and measures the ability of a basis to represent PW eigenstates, by measuring how much of the subspace of the Hamiltonian eigenstates falls outside the subspace spanned by the atomic basis. If we consider the PW eigenfunctions $\left|\psi_{\alpha}(\mathbf{k})\right\rangle$ and their projection into the atomic basis $P(\mathbf{k})\left|\psi_{\alpha}(\mathbf{k})\right\rangle$, then $\mathcal{S}_{\Omega}$ gives the average of $\|(1-$ $P)\left|\psi_{\alpha}(\mathbf{k})\right\rangle \|^{2}$ over the eigenstates considered for the projection. It must be also pointed out that the projected eigenstates $\left|\chi_{\alpha}(\mathbf{k})\right\rangle=P(\mathbf{k})\left|\psi_{\alpha}(\mathbf{k})\right\rangle$ are not necessarily orthonormal, the overlap matrix $R_{\alpha \beta}(\mathbf{k})=\left\langle\chi_{\alpha}(\mathbf{k}) \mid \chi_{\beta}(\mathbf{k})\right\rangle$ 
being different from the identity matrix. In particular $R_{\alpha \alpha}(\mathbf{k}) \leq 1$, and

$$
\mathcal{S}_{\Omega}=\frac{1}{N_{k}} \frac{1}{N_{\alpha}} \sum_{k}^{N_{k}} \sum_{\alpha=1}^{N_{\alpha}}\left(1-R_{\alpha \alpha}(\mathbf{k})\right)
$$

is the averaged norm which has been lost (spilled) in the projection process. If we restrict our analisys to the occupied eigenstates, then $\mathcal{S}_{\Omega}$ is the fraction of total electronic charge spilled when projecting, and will be refered to as charge spilling $\mathcal{S}_{Q}$.

A local spilling can also be defined as a function of real space coordinates,

$$
\mathcal{S}_{\Omega}(\mathbf{r})=\frac{1}{N_{k}} \frac{1}{N_{\alpha}} \sum_{k}^{N_{k}} \sum_{\alpha=1}^{N_{\alpha}}\left(\left|\psi_{\alpha}(\mathbf{k}, \mathbf{r})\right|^{2}-\left|\chi_{\alpha}(\mathbf{k}, \mathbf{r})\right|^{2}\right)
$$

We consider it a useful and direct way for visually characterizing the quality of the representation of the PW eigenstates. The total spilling defined above is recovered by integrating the local spilling over the three-dimensional space.

Important for the characterization of the quality of a basis for a given system is the energy band structure. To calculate the LCAO energy bands of a system we project the Hamiltonian associated to the self-consistent PW charge density of our reference calculation into the basis subspace:

$$
H_{\mu \nu}^{L C A O}(\mathbf{k})=\left\langle\phi_{\mu}(\mathbf{k})\left|H^{P W}\right| \phi_{\nu}(\mathbf{k})\right\rangle
$$

To obtain the LCAO Hamiltonian matrix elements we expand the Bloch basis functions into plane waves $\mathrm{We}$ calculate the kinetic energy and the effect of the non-local part of the pseudopotential directly in Fourier space. The effect of the local part of the potential and self-consistent Hartree and LDA interactions is calculated using fast Fourier transform algorithms,

$$
\begin{gathered}
H_{\mu \nu}^{L C A O}(\mathbf{k})=\sum_{|\mathbf{k}+\mathbf{G}|^{2}<E_{\max }}\left\langle\phi_{\mu}(\mathbf{k}) \mid \mathbf{k}+\mathbf{G}\right\rangle\left\langle\mathbf{k}+\mathbf{G}\left|H^{P W}\right| \phi_{\nu}(\mathbf{k})\right\rangle \\
S_{\mu \nu}^{L C A O}(\mathbf{k})=\sum_{|\mathbf{k}+\mathbf{G}|^{2}<E_{\text {max }}}\left\langle\phi_{\mu}(\mathbf{k}) \mid \mathbf{k}+\mathbf{G}\right\rangle\left\langle\mathbf{k}+\mathbf{G} \mid \phi_{\nu}(\mathbf{k})\right\rangle
\end{gathered}
$$

where $\mathbf{G}$ are reciprocal lattice vectors and $E_{\max }$ is an energy cut-off independent of the one used in the PW calculation, that must be large enough to guarantee a reliable representation of the atomic basis. With this method we obtain a LCAO Hamiltonian from a first-principles PW calculation, no free parameters being fitted.

The LCAO Hamiltonian is obtained in Bloch space and, as a consequence, it includes interactions up to infinite neighbors. The deviations in the band structure with respect to the $\mathrm{PW}$ only reflect the uncompleteness and inadequacy of the basis, clearly separated from the possible additional approximation of neglecting matrix elements beyond some range of interactions. We can study both approximations separately. In most studies about LCAO band structures these two effects are mixed together. Af an exception it must be mentioned the works of Chadid and Jansen and Sankey, 14 where also LCAO Hamiltonians with interactions up to an infinite range of neighbors are obtained.

The Hamiltonian matrix in real space is calculated from the Hamiltonian matrix in Bloch space by performing a Bloch type transformation

$$
\begin{aligned}
& H_{\mu \nu}^{L C A O}\left(\mathbf{R}_{\mu \nu}\right)=\sum_{\mathbf{k}} H_{\mu \nu}^{L C A O}(\mathbf{k}) e^{i \mathbf{k}\left(\mathbf{R}_{\mu}-\mathbf{R}_{\nu}\right)} \\
& S_{\mu \nu}^{L C A O}\left(\mathbf{R}_{\mu \nu}\right)=\sum_{\mathbf{k}} S_{\mu \nu}^{L C A O}(\mathbf{k}) e^{i \mathbf{k}\left(\mathbf{R}_{\mu}-\mathbf{R}_{\nu}\right)}
\end{aligned}
$$

where normalization factors which depend on the overlaps are omitted for clarity. The sum has to be extended to a sufficient number of $\mathbf{k}$ points, taking into account that the number of pqints depends on the real space range of the interactions. 15.16

The projection technique can also be used for obtaining chemical information from the PW calculations by means of an LCAO population analisys. We use the one proposed by Mulliken.17 The PW occupied eigenstates are projected onto the subspace spanned by the atomic basis. Due to the non-orthogonality of the projected eigenstates, the density operator has to be properly defined to ensure the charge conservation

$$
\hat{\rho}=\sum_{\mathbf{k}} \sum_{\alpha}^{o c c}\left|\chi_{\alpha}(\mathbf{k})\right\rangle\left\langle\chi^{\alpha}(\mathbf{k})\right|
$$

where $\left|\chi^{\alpha}(\mathbf{k})\right\rangle=\sum_{\beta} R_{\beta \alpha}^{-1}(\mathbf{k})\left|\chi_{\beta}(\mathbf{k})\right\rangle$ represent the vectors of the dual set of the projected eigenstates, and $R_{\beta \alpha}(\mathbf{k})=\left\langle\chi_{\beta}(\mathbf{k}) \mid \chi_{\alpha}(\mathbf{k})\right\rangle$. The LCAO density matrix is then written in terms of the dual LCAO basis:

$$
\mathcal{P}_{\mu \nu}=\left\langle\phi^{\mu}|\hat{\rho}| \phi^{\nu}\right\rangle,
$$

the charge associated to an orbital $\mu$ being

$$
Q_{\mu}=\sum_{\nu} \mathcal{P}_{\mu \nu} S_{\nu \mu}
$$

\section{REFERENCE CALCULATIONS}

The analysis of the bases presented in this paper are based on reference PW calculations which have been performed using ab initio pseudopotentials generated by the improved Troullier-Martins method 18 and within 
the local density approximation (LDA) for electron exchange and correlation.19 Of course, other schemes could have been equally used, the only requirement to apply the method being the planewave expansion of the oneelectron wavefunctions. The energy cut-off for the planewave expansion has been taken large enough to consider that all the calculations are well converged. High energy cut-offs are not necessary for the application of the method but are important to ensure accurate results for the optimization of the orbitals as well as for the population analisys. The cutoffs used are: $10 \mathrm{Ry}$ for $\mathrm{Al}$, $20 \mathrm{Ry}$ for $\mathrm{Si}$, BP, and AlP, 30 Ry for GaAs, 40 Ry for graphite, $50 \mathrm{Ry}$ for $\mathrm{BN}$, and $70 \mathrm{Ry}$ for $\mathrm{C}$ and $\mathrm{SiC}$. For the self-consistent zincblende semiconductor calculations, we used 2 irreducible-Brillouin-zone points (IBZP), equivalent to 32 whole-Brillouin-zone points (BZP), giving a $\mathrm{k}$-mesh length cutofft $l_{c}=a$, where $a$ is the lattice constant. For aluminum and graphite we used respectively (10 IBZP, $125 \mathrm{BZP}, l_{c}=13.435$ a.u.) and (8 IBZP, 16 $\mathrm{BZP}, l_{c}=4.648$ a.u.). The length cutoff $l_{c}$ is simply related with the maximum range of the real-space matrix elements obtainable from the PW calculation (Eqs. 11 and 12) by $\left|\mathbf{R}_{\mu \nu}\right|<l_{c}$. For the pseudopotentials, in all the calculations we have used the separable, fully nnlocal formulation following Kleinman and Bylander 20

\section{ATOMIC BASIS SETS}

A brief description follows of the sets of atomic orbitals which are used throughout this work. The studied basis sets can be classified in two groups: (i) those which are provided by the numerical solution of the atomic problem (with the same atomic pseudopotential as used in the PW calculatipn), hereafter called PAO's for pseudo atomic orbitals, 13,4 and (ii) Slater-type orbitals (STO).8

Within the first group, $\mathrm{PAO}_{\infty}$ are the atomic solutions under real atomic boundary conditions (at $r=\infty$ ). This family of bases allows for a scaling factor for the radial part acting as a variational parameter to optimize the atomic orbitals via the spilling minimization,

$$
\phi_{l}^{o p t}(\mathbf{r})=\lambda_{l}^{\frac{3}{2}} \phi_{l}^{a t m}\left(\lambda_{l} \mathbf{r}\right) .
$$

$\mathrm{PAO}_{\infty}$ represents quite a natural choice, since access to $\mathrm{PW}$ calculations is usually accompanied by access to atomic calculations under the same approximations. They provide a good description of the charge density and have already been used in $a b$ initio calculations 14 giving accurate results. For covalent materials, with small charge transfers, they provide a very good description of the pseudo-core region. This is worth considering since the PW eigenstates are expanded in the atomic basis maximizing the projection without making any distinction between the pseudo-core region (zone near the atomic nucleus) and the valence region (interatomic zone).
The main disadvantage of the $\mathrm{PAO}_{\infty}$ 's is the very long range of the interactions and overlaps they originate. Getting a shorter and perfectly defined range of interactions is the reason to work with $\mathrm{PAO}_{r_{c}}$. They are the solutions of the atomic pseudopotential when forced to vanish outside a cutoff radius $r_{c}$, keeping the continuity, as introduced by Sankey and Niklewski 13 (they will be denoted by specifying the value of $r_{c}$ in atomic units). The cutoff radius represents a variational parameter for this type of bases. These orbitals seem promising candidates in calculation techniques for very large systems.

Within the Slater orbitals, we call STO1 the conventional orbitals $r^{n-1} \exp ^{-\beta r}$, where $n$ is the principal quantum number and the scale factor $\beta$ is taken to be the same for all the atomic-orbitals within the same shell; STO2 will be the same, but allowing for a different $\beta$ for each orbital, $\beta_{s} \neq \beta_{p} \neq \beta_{d}$; and STO3 are the orbitals $r^{\alpha} \exp ^{-\beta r}$, where $\alpha$ becomes another variational parameter, no longer restricted to be an integer, and allowing both $\alpha$ and $\beta$ to be different for each orbital.

STO's have been extensively used in LCAO calculations in quantum chemistry and have proven to provide accurate results. They present the additional advantage of their very simple mathematical properties. To simplify the computational task, it is a common practice to expand the STO's orbitals as linear combinations of gaussian functions. These are the so-called STO-NG5 orbitals where $\mathrm{N}$ stands for the number of gaussians orbitals used per STO. We have done some tests to compare the results obtained using STO and STO-4G orbitals. No appreciable differences have been found in the results. Spilling values and optimum exponents are essentially the same in both cases, and no further investigation has been pursued concerning STO-NG.

STO orbitals do not seem a priori to be very well suited for the description of the pseudo-core region for the projection, possibly leading to worsen the spilling at the valence region while trying to adapt the pseudo-core. To explore that possibility we consider a last kind of orbital functions, the pseudo-STO, which represent a link between the STO and the PAO families. A pseudo-STO is a Slater-type orbital outside a certain radius, and the numerical solution of the pseudopotential inside that radius, continuity of the function and its derivative being imposed.

\section{SPILLING ANALYSIS}

This section is devoted to show the adequacy of an analysis based on the projection and the spilling for the evaluation of a basis set. The total spilling provides a natural parameter that can be used straightforwardly to measure systematically the quality of a basis. The optimization in terms of spilling provides a variational scheme, since the minimum attainable is zero, which means a perfect basis. 
Both the projected charge density and the local spilling offer a useful tool for visually characterizing the quality of a basis. This is illustrated in Fig. 1, where a charge-density contour map for $\mathrm{SiC}$ is shown comparing the $\mathrm{PW}$ results with the results of the projection on the $\mathrm{PAO}_{\infty}$, showing in addition the local spilling along the bond axis. We see that some of the bond structure is lost, the charge density being much more spherical around the atoms, when projecting on this basis. This can be also seen in the fact that the two maxima in the density near the carbon nucleus are of approximately the same height, while in the PW calculation there is more density at the bond side. All these features can be seen in the local spilling, which is negative in the regions with an excess of projected charge and positive in the zones with a defect of projected charge. The local charge spilling is negative in the proximity of both core regions and positive in the bond region. Some of the bond charge goes to an anti-bonding region. This feature also tends to make the charge-transfer lower for this $\mathrm{PAO}_{\infty}$ basis (see below).

For the spilling analysis to be useful, the spilling has to be correlated with the quality in the energetics of the system. This is tested in Fig. 2, where the mean square error of the projected valence bands of crystralline silicon, as compared to the PW ones, is plotted versus the charge-spilling $\mathcal{S}_{Q}$, for different families of bases. It is calculated without shifting the bands, the absolute zero being unambiguously defined (the Hamiltonian operator is not changed). This quantity can also be taken as an indicator of the error in the total energy due to the approximation of the basis.

The clear correlation between the spilling and the error in the energy bands displayed in Fig. 2 indicates that a basis optimization based on a minimization of the spilling is parallel (at least approximately) to an energy minimization. This parallelism is even clearer within a basis family, where the correlation is much more pronounced (different points within the same family are obtained by varying the characteristic parameters presented above).

To finish this section, we introduce a lower bound for the spilling. The dependence of $\mathcal{S}_{\Omega}$ on the atomic basis enters through the Fourier coefficients of the radial wavefunctions. Therefore, $\mathcal{S}_{\Omega}$ can be regarded as a function of these Fourier coefficients $\phi_{i, \mu}(|\mathbf{k}+\mathbf{G}|)$ which are different for each atom $i$, orbital $\mu$ and each modulus $|\mathbf{k}+\mathbf{G}|$. Considering all these Fourier coefficients as free varables the minimization of $\mathcal{S}_{\Omega}$ gives a lower bound for $\mathcal{S}_{\Omega}$. Values of this lower bound will be shown as Min. $\mathcal{S}$ in the tables.

\section{ANALYSIS AND OPTIMIZATION OF ATOMIC BASIS SETS}

The quality of the LCAO bases presented above is measured and optimized for different solids in this section using the spilling analysis, and, simultaneously, the LCAO band structure is contrasted with the results of planewaves. The results are displayed in Tables I through IV. The resulting numbers are abundant since the number of possibilities is large. For the different kinds of bases introduced in section IV there are different possible basis sizes, single- $z$ or double- $z$, meaning one or two orbitals per atomic symmetry. There is the additional possibility of polarizing the basis, i.e., introducing atomic orbitals of a higher angular momentum than needed, which, in the cases of this paper represents the addition of the $d$ orbitals to the minimal $s p$ basis.

The optimization of the different bases introduces new degrees of freedom since their optimization is done with respect to different variational parameters (see section IV), and also the spilling can be defined for different numbers of bands, depending on the particular application the basis is optimized for.

\section{A. Minimal basis sets}

Table I shows the spillings of the projections onto different minimal bases for silicon, diamond, and aluminum, together with the mean square error in the LCAO bands as compared with the PW ones. Only $s p$ bases are considered, except for $\mathrm{Al}$, for which also spd bases data are shown due to the poorness of minimal $s p$ bases for this solid. The numbers displayed for STO bases have been obtained after minimization of the charge spilling (the spilling of the valence eigenvectors) for the corresponding parameters. This procedure is illustrated in Fig. 3 for the specific cases for silicon and carbon, where the charge spilling is shown as a function of the STO exponents. $\mathrm{PAO}_{\infty}^{\text {opt }}$ stands in Table I for the $\mathrm{PAO}_{\infty}$ optimized via a scale factor. The $\mathrm{PAO}_{r_{c}}$ are optimized with respect to the radius of the basis functions, $r_{c}$, as illustrated in Figs. 4, 5, and 6 for silicon, diamond, and aluminum, respectively. In Table I, especific values of $r_{c}$ are chosen.

All the bases displayed in Table I perform comparably well in what concerns the charge spilling. Clearly better does $s p$ for $\mathrm{C}$ than for $\mathrm{Si}$ and $\mathrm{Al}$, as expected since $d$ orbitals play a lesser role for $\mathrm{C}$ than for $\mathrm{Si}$ and $\mathrm{Al}$. The spilling for eigenstates including conduction bands is larger than for valence bands only. For $s p$ bases there is a factor of 10 to 20 . The two main reasons for that are, (i) the bases have been optimized by minimizing the charge spilling, and (ii) higher energy bands require higher energy atomic orbitals.

Table I also shows the populations of $s, p$, and $d$ orbitals (if present). It can be seen that the $s p^{3}$ hybridization is better accomplished in $\mathrm{C}$ than in $\mathrm{Si}$ (a perfect hybridization would show $Q_{s}=1$ and $\left.Q_{p}=3\right)$. $Q_{d}$ in $\mathrm{Al}$ is quite appreciable.

The mean square error in the energy bands is displayed as $\sigma_{v}$ for the valence bands and $\sigma_{c}$ for the first two conduction bands $\left(\sigma_{1+2}\right.$ for first two bands, and $\sigma_{3+4}$ for third and forth, in the case of aluminum). The valence 
bands are quite well described with $s p$ bases for Si and C, but not for $\mathrm{Al}$. The metallicity in $\mathrm{Al}$ demands a larger contribution of $d$ orbitals, what makes them necessary for any quantitative LCAO description of Al. The conduction bands are much worse described by the minimal bases shown in the table.

The dependence in the number of bands to be optimized through the spilling is shown in Table II for $\mathrm{Si}$, $\mathrm{C}, \mathrm{BN}$, and Al. Each kind of basis has been optimized by minimizing (i) the charge spilling and (ii) a spilling including conduction bands $\left(\mathcal{S}_{8}\right.$ for $\mathrm{Si}, \mathrm{C}$, and $\mathrm{BN}$, and $\mathcal{S}_{4}$ for $\left.\mathrm{Al}\right)$. It can be seen how an improvement in $\mathcal{S}_{8(4)}$ is accomplished when optimizing the basis for it, but paying the prize in the worsening of $\mathcal{S}_{Q}$. In addition to the $\mathcal{S}$ and $\sigma$ values, the band gap region description is evaluated by showing the values of the $\Gamma$-direct and indirect gaps, and the position of the minimum of the conduction band. An important and systematic improvement is observed in these quantities when the optimization is extended to unoccupied states. The displayed information offers the necessary information for the evaluation of the trade-off in the quality of the different spectral regions for the choice of a basis for a particular application. Fig. 7 shows the band structure of Si obtained with a minimal $s p$ STO3 basis optimized considering $\mathcal{S}_{8}$. Notice the nice agreement in the band-gap region, showing a very realistic indirect gap, quite rare for a minimal basis [compare with the typical cases shown in Fig. 8 (a) and (d)]. The LCAO bands have been shifted by $1.04 \mathrm{eV}$, what means an important deviation in total energies, if computed. Another price to pay is the very long range interactions due to the unusual extension acquired by the orbitals in the optimization.

Trying to improve the performance, other, more complicated, kinds of bases have also been tried, the results not being shown in the Tables. Pseudo-STO's (see section IV) provide good bases, but the improvement on the others (STO and PAO) have not been substantial enough for seriously considering them as alternative candidates. They have been optimized by varying the STO parameters outside the core region (as for STO3) plus the extra degree of freedom given by the core radius. For Si a minimum in $\mathcal{S}_{Q}$ is obtained for a core radius of 1 a.u., but the spilling is only $4 \%$ lower than the spilling of the STO3 case. This fact indicates that the projection is not as sensitive to the core regions so as to worsen the overall projection if the core region in the basis orbitals is not adapted to the pseudopotential.

Another kind of basis has been constructed based on $\mathrm{PAO}_{r_{c}}$. More degrees of freedom are given to the spilling minimization by linear combining excited states of the atomic problem to the ground state. For each symmetry $\left(s, p_{x}, p_{y}, p_{z}, \ldots\right)$ the atomic orbital is constructed with a linear combination of the ground and excited states of the corresponding pseudopotential. This procedure considerably reduces the spilling without increasing the size of the basis. However, the band structure features are not accordingly improved. This fact leads to the conclu- sion that, for the spilling-energy correlation to be satisfactory in the solid, the energetics in the basis itself (in the atomic problem) has to be considered.

\section{B. Range of interactions}

An important aspect defining the applicablity of LCAO bases is their range of interactions, i.e., the scope of neighbor shells that have to be included in the LCAO Hamiltonian and overlap matrices. The basis sets giving comparable results in the previously shown quantities perform quite differently in this respect. This is shown in Table III. This information is obtained by computing the atomic matrix elements of the Hamiltonian and overlap matrices as described in section II. Due to the different analytical expressions for the orbitals, to measure the interaction range we adopt an empirical criterium, namely, the radius $r_{99.9 \%}$ for which $99.9 \%$ of the norm of the orbital is within the sphere with that radius. We have found that criterium meaningful in the sense that neglecting interactions beyond that radius does not appreciably alter the energy bands corresponding to the valence and some conduction bands (average error lower than 0.05 $\mathrm{eV}$ ). Neglecting interactions within that radius produces appreciable deviations in the bands. For $\mathrm{PAO}_{r_{c}}$ 's the interacting scope is clearly defined by $r_{c}$ (which incudes $100 \%$ if the norm within), but for short $r_{c}$ 's they are very similar to $r_{99.9 \%}$, and for larger $r_{c}$ 's then $r_{99.9 \%}$ becomes more meaningful. Therefore, we also characterize the $\mathrm{PAO}_{r_{c}}$ 's with $r_{99.9 \%}$. Besides this quantity, Table III also shows the scope of non-negligible neighboring shells for each case.

$\mathrm{PAO}_{\infty}$ represents the best basis in what spilling and band quality is concerned, and also for population purposes 9 (see next section), but its range of interactions is very large. This fact was already pointed out by Jansen and Sankey 14 in their work doing self-consistent calculations using this basis. The results of our calculations show that interactions up to at least 5 (10) shells of neighbors have to be kept for $\mathrm{Si}(\mathrm{C})$ to obtain a reasonable band structure with the correct semiconducting behavior. The best basis suited for a shorter range of interactions together with keeping good standards in spilling and band structure is the $\mathrm{PAO}_{r_{c}}$, as can be seen in Table III and Fig. 4 for Si, in Fig. 5 for C, and in Fig. 6 for Al. Note that, in spite of $\mathrm{C}$ needing a smaller $r_{c}$ than $\mathrm{Si}$ to attain small spillings, the range of neighbors is larger for $\mathrm{C}$ due to the much smaller lattice parameter.

\section{Polarized and double- $z$ basis sets}

The clearest way of improving the quality of a basis is increasing its size. In LCAO there are two usual ways of doing it, (i) increasing the number of orbitals within the same atomic symmetries as found in the minimal basis, 
and (ii) polarizing the basis. Within the first, double$z$ is the most usual case, i.e., doubling the basis, which in the cases of this paper means a basis of two $s$, two $p_{x}$, two $p_{y}$ and two $p_{z}$ orbitals. A polarization of the basis is accomplished when a shell of different atomic symmetry is added to the minimal basis. In our cases it means the addition of the $d$ shell to the minimal $s p$ basis. Combination of these two procedures is also customary for further improvement. A rule of thumb in quantum chemistry says that a basis should always be doubled before being polarized.5 This is not the usual practice within the LCAO calculations in the solid-state-physics community, where double- $z$ bases are hardly to be found

Expanded STO bases can be found in the literature.5 This is not the case for PAO's. In this paper we propose to use the excited states of the atomic calculations leading to the $\mathrm{PAO}_{r_{c}}$ 's, under the same boundary conditions, to obtain the orbitals required to expand the basis, i.e., excited $s$ and $p$ orbitals for double- $z$ bases and $d$ orbitals for polarization. The advantages of this scheme are its simplicity and the controlled range of interactions also for the added orbitals, which is usually lost in the traditional scheme.

Again, we study here the quality of expanded LCAO bases by means of their spilling and their band structure as obtained from the projection. The results of our analysis are shown in Table IV, where minimal (single- $z$ ), double- $z$, and polarized single- $z$ bases are compared for silicon and diamond. See also Table I for aluminum. The sp and spd bases are optimized as above using STO3 for the Slater orbitals, and using the corresponding $r_{c}$ for the PAO's, as shown in Figs. $4-6$ Double- $z$ for STO has been taken from the literature 5,21 Note that, with double- $z, \mathrm{Si}$ does not reach equivalent quality as $\mathrm{C}$, and that, for that purpose, the polarization of the basis is quite enough.

The band structures corresponding to the data of Table IV are shown in Fig. 8 for Si, Fig. 9 for C, and Fig. 10 for Al (Table I), in each case comparing the LCAO bands obtained from the projection with the reference PW bands. The information given in the Table is ratified, and observed in more detail. Note that the substantial improvement in the Si case occurs when including the $d$ orbitals, but not when doubling the minimal basis. For diamond, however, such qualitative improvement is given by the doble- $z$ basis. The figures for the gap in the $\mathrm{Si}$ and $\mathrm{C}$ cases are shown in Table $\mathrm{V}$. In the $\mathrm{Al}$ case, the polarization is needed from the start, the $s p$ projection giving defective band structures in the neighborhood of the Fermi level around the $\mathrm{W}$ point. The spd band structure is shown in Fig. 10 for $\mathrm{PAO}_{r_{c}}$ for different $r_{c}$. The performance of the double and polarized STO bases in the first conduction bands is better than that of the corresponding $\mathrm{PAO}_{r_{c}}$ bases. This is due to the larger extension of the extra orbitals in the STO bases, which is not allowed in the $\mathrm{PAO}_{r-c}$ 's. The latter, however, also represent a considerable improvement over the minimal bases, while keeping the interaction range. This virtue makes them very appealing for accurate LCAO calculations.
The error in the band structures could be noticeably reduced with a rigid shift of the bands, which is clear in sight of the Figures. This has not been allowed in order to keep the band-structure error as representative of the behavior to be expected of total energies.

\section{Geometry and environment dependence}

The projection procedure proposed in this paper is based on PW calculations of actual materials. As compared to basis optimization in atoms, this allows adequating the basis to particular environments or geometries. As a first example we show the change in the minimal $s p \mathrm{PAO}_{r_{c}}$ basis for $\mathrm{C}$ depending on the solid being diamond or graphite. Fig. 11 shows the charge spilling as a function of $r_{c}$ and of the range of interacting neighboring shells. Graphite is worse represented by the same kind of basis. In addition, graphite requires larger $r_{c}$ to approach minimum spilling. This latter fact is due to the comparatively large interplanar distances in the graphite structure.

Another example of geometry dependence of the basis quality is given in Fig. 12, where the charge spilling of a minimal $\mathrm{PAO}_{r_{c}}$ for $\mathrm{Si}$ is plotted versus the lattice constant for different values of $r_{c}$. From the Figure it is clear that (i) for spilling purposes and, therefore,for the total energy, the basis is better the larger $r_{c}$, (ii) the slope in $\mathcal{S}_{Q}$ versus lattice constant will translate in a corresponding slope of the total energy, what originates a fictitous pressure on the system, (iii) this pressure is different depending on $r_{c}$, and the optimum $r_{c}$ for this purpose (the one giving zero pressure, with $r_{c}$ between 5 and 6 a. u.) is different from the optimum for energy considerations $\left(r_{c}=\infty\right)$, and (iv) there are no appreciable curvatures, from where it can be inferred that no substantial deviations are to be expected in the ellastic and force constants (vibrations) due to the basis.

\section{POPULATION ANALYSIS}

One of the uses of the projection into LCAO is the possibility of performing population analysis of the $\mathrm{PW}$ results, otherwise inaccesible. It is well known that any population analisys has an inherent arbitraryness associated to the fact that the relevant quantities in the analysis do not correspond to observables, but rather to convenient ways of dissecting the total charge of the system. Furthermore, small variations in the basis can produce quite different population results, particulary for charge transfers. In spite of it, much of the chemical descriptions of condensed systems is performed in the language given by such population analyses. We, therefore, consider it worthwhile to explore the problem with the spilling.

Due to the mentioned arbitraryness, the correlation of charge transfers and charge spilling is expected to be 
poor. This is clear when uncompensating the basis, like, for instance, when introducing $d$-orbitals for silicon in $\mathrm{SiC}$. This greatly improves the description of the eigenfunctions and reduces the spilling, but being the basis uncompensated, some features (structure of the bond) are described by the much more complete basis of one of the atoms, so that more electronic charge is assigned to the atom with more complete basis, getting an unphysical charge transfer. A compensated basis is therefore important for the populations to be meaningful. A measure of this compensation can be obtained if the spilling is calculated independently for structurally similar solids containing the individual atoms separately. The spillings for silicon and diamond separately, using the same kind of basis for both, indicate a slight uncompensation favoring diamond, which, in this case says that the charge transfer might be slightly overestimated. In addition to that, the optimization of the basis is important to get reliable values of charge transfers. This is seen in Fig. 13, where the charge transfer in $\mathrm{SiC}$ is shown to correlate with the spilling. A 'converged' charge transfer is not guaranteed, but, provided that the basis is compensated, a small spilling indicates a meaningful charge transfer.

Table VI shows the charge transfers for some zincblende semiconductors, obtained with the projection procedure, using optimized $\mathrm{PAO}_{\infty}$ basis sets. The numbers compare well with equivalent results $q$ tained from direct LCAO selfconsistent calculations.22,23 Systems with only one species do not present these difficulties for the charges since the basis can be trivially compensated. Table VII shows the excess of charge (with respect to the neutral atom) assigned to the upper and lower atoms of a dimer in the $\operatorname{Si}(100)-2 \times 1$ surface $24 \mathrm{~A}$ minimal basis has been used. The populations are quite stable and independent of the basis used.

\section{CONCLUSIONS}

We have presented a projection method which provides a tool to characterize and optimize atomic orbital basis sets for a given system. Independently of how many bases are evaluated for that particular system, only one reference self-consistent planewave calculation is required. The method also provides LCAO band structures, Hamiltonian matrix elements (non fitted tight-binding parameters), and population analysis.

Based on that tool and on its characteristic parameter, the spilling, an exhaustive analysis of two kinds of LCAO basis sets, namely, Slater-type (STO) and pseudoatomic (PAO) orbitals, have been presented for several solids, arriving to the following conclusions:

(i) The spilling provides a very convenient tool for studying and variationally optimizing basis sets, that closely correlates with energy optimizations.

(ii) The LCAO band structures obtained from the projection allow to characterize separately the errors due to the uncompleteness of the basis and to considering a limited range of interactions. This is due to the fact that the bands are obtained directly in Bloch space, with interactions to infinite neighbors.

(iii) Both STO's and PAO's optimized with spilling minimization give quite good basis sets, of comparable quality.

(iv) If a controlled range of neighbor interactions is desired, the best performing basis sets are provided by the $\mathrm{PAO}_{r_{c}}$ introduced by Sankey and Niklewski.13

$(v)$ A simple and interaction-range-controlled scheme for extending PAO's basis sets to double- $z$ and/or polarized bases is proposed, and shown to give good basis sets.

(vi) Double- $z$ bases substantially improve the performance on diamond, whereas $d$ polarization is required for similar results in $\mathrm{Si}$ and $\mathrm{Al}$.

(vii) The spilling minimization allows optimization taylored for particular uses, like for the description of excitations or band gaps, environment dependence of the basis, etc.

(viii) Population analysis can be performed, and its intrinsic arbitraryness can be partially controlled by means of the spilling.

\section{ACKNOWLEDGMENTS}

We acknowledge useful discussions with F. Ynduráin and P. Ordejón. This work has been supported by the Dirección General de Investigación Científica y Tecnológica of Spain (DGICYT) under grant PB92-0169.

${ }^{1}$ H. Eschrig, 'Optimized LCAO method', Spriger-Verlag (1987), and references therein.

2 See, for instance, J. Ihm, A. Zunger, and M. L. Cohen, J. Phys. C: Solid St. Phys. 12, 4409 (1979).

${ }^{3}$ D. R. Hamann, M. Schlüter, and C. Chiang, Phys. Rev. Lett. 43, 1494 (1979).

${ }^{4}$ P. Ordejón, D. A. Drabold, R. M. Martin, and M. P. Grumbach, Phys. Rev. B 51, 1456 (1995), and refereces therein.

${ }^{5}$ R. Poirier, R. Kari, and I. G. Csizmadia, 'Handbook of gaussian basis sets', Elsevier Science (1985), and references therein.

${ }^{6}$ D. J. Chadi, Phys. Rev. B 16, 3572 (1977).

${ }^{7}$ E. Francisco, L. Seijo, and L. Pueyo, J. Solid State Chem. 63, 391 (1986), and references therein.

${ }^{8}$ J. M. García de la Vega, J. Fernández Rico and J. I. Fernández-Alonso, J. Mol. Struct. (Theochem), 210, 79 (1990).

${ }^{9}$ D. Sánchez-Portal, E. Artacho, and J. M. Soler, Solid State Commun. 95, 685 (1995).

${ }^{10}$ P. Ordejón and F. Ynduráin, Phys. Rev. B 43, 4552 (1991). 
${ }^{11}$ E. Artacho and F. Ynduráin, Phys. Rev. B 44, 6169 (1991).

${ }^{12}$ E. Artacho and L. Miláns del Bosch, Phys. Rev. A 43, 5770 (1991).

${ }^{13}$ O. F. Sankey and D. J. Niklewski, Phys. Rev. B 40, 3979 (1989).

${ }^{14}$ R. W. Jansen and O. F. Sankey, Phys. Rev. B 36, 6520 (1987).

${ }^{15}$ H. J. Monkhorst and J. D. Pack, Phys. Rev. 13, 5188 (1976).

16 J. Moreno and J. M. Soler, Phys. Rev. B 45, 13891 (1992).

${ }^{17}$ R. S. Mulliken, J. Chem. Phys. 23, 1833 (1955).

${ }^{18}$ N. Troullier and J. L. Martins, Phys. Rev. B 43, 1993 (1991).

19 P. Hohenberg and W. Kohn, Phys. Rev. 136, B864 (1964); W. Kohn and L. J. Sham, Phys. Rev. 140, A1133 (1965).

${ }^{20}$ L. Kleinman and D. M. Bylander, Phys. Rev. Lett. 48, 1425 (1982).

${ }^{21} \mathrm{P}$. Ordejón, private communication: basis $14-20-1$ from Ref. 5 slightly modified for bulk calculations has been used for $\mathrm{Si}$, and a contraction of the basis proposed by van Duijneveldt (IBM Res. Reports RJ945, 1971) for diamond.

${ }^{22}$ R. Orlando, R. Dovesi, C. Roetti, and V. R. Saunders, J. Phys. : Condens. Matt. 2, 7769 (1990).

${ }^{23}$ J. R. Chelikovsky and J. K. Burdett, Phys. Rev. Lett. 56, 961 (1986)

${ }^{24}$ Geometry taken from J. M. Soler (unpublished).

FIG. 1. Electron charge density for SiC: (a) self-consistent density contour map in the (110) plane calculated with PW, (b) the same after projecting the eigenstates into a $\mathrm{PAO}_{\infty} s p$ basis, and (c) these two densities (PW with solid lines and the projected density with dotted lines) and $\mathcal{S}_{Q}(\mathbf{r})$ along the bond axis. Units are electrons per unit cell.

FIG. 2. Mean square deviation in valence bands of the projected hamiltonian from the PW bands versus the charge spilling of different bases in silicon. Each symbol stands for a different family of bases: $\square$ for $\mathrm{PAO}_{r_{c}}, \times$ for $\mathrm{PAO}_{\infty},+$ for STO, and $\diamond$ for different double- $z$ bases. Bases with $\sigma_{v}$ lower than $0.2 \mathrm{eV}$ include $d$ orbitals.

FIG. 3. Charge spilling versus exponent for a STO1 basis set, (a) silicon with a $s p d$ basis, (b) diamond with a $s p$ basis. Also shown the optimum exponents for a STO2 basis set for the same systems.

FIG. 4. (a) Charge spilling for silicon with a $\mathrm{PAO}_{r_{c}} s p$ and $s p d$ basis versus $r_{c}$, (b) charge spilling versus the shell of neighbors with non-zero overlaps for the spd basis, and (c) for the $s p$ basis.

FIG. 5. (a) Spilling for occupied and first eight bands for diamond with a $\mathrm{PAO}_{r_{c}} s p$ versus $r_{c}$, (b) charge-spilling versus the shell of neighbors with non-zero overlaps basis, (c) the same for the spilling of the first eight bands.
FIG. 6. The same as Fig. 4 for aluminum.

FIG. 7. Gap optimization with a minimal basis. Silicon band structure calculated with a STO3 sp optimized to reproduce the occupied eigenstates and the first four conduction bands. The PW band structure is shown with dots. The STO3 bands have been shifted $1.04 \mathrm{eV}$ to make the top of both valence bands coincide.

FIG. 8. Band structure of Si for minimal, double- $z$, and $s p d$ bases. In (a), (b) and (c) the Hamiltonian is projected in a STO3 $s p$, STO double- $z$, and STO3 spd basis, respectively. STO3 bases optimized for valence band. (d),(e) and (f) correspond to $\mathrm{PAO}_{5.0} s p$, double- $z$, and $s p d$ basis. The PW reference band structure is shown with dots.

FIG. 9. Band structure of diamond for single- $z$ and double- $z$ bases. (a) and (b) correspond the projection in a STO3 $s p$ and STO double- $z$ basis sets, respectively. STO3 basis optimized for valence band. Figures (c) and (d) correspond to $\mathrm{PAO}_{4.0} s p$ and double- $z$. The PW reference band structure is shown with dots.

FIG. 10. Aluminum band structure: (a) calculated with $\mathrm{PAO}_{5.3}$ spd basis, and (b) using $\mathrm{PAO}_{6.1}$. Dotted lines show the PW band structure (Fermi energy is $8.2 \mathrm{eV}$ ).

FIG. 11. (a) Charge spilling for diamond (D) and graphite $(\mathrm{G})$ as a function of $r_{c}$ for a $\mathrm{PAO}_{r_{c}} s p$ basis. (b) Charge spilling for diamond as a function of the shell of neighbors with non-zero overlap, and (c) the same for graphite.

FIG. 12. (a) $\mathcal{S}_{Q}$ versus lattice constant of silicon for $\mathrm{PAO}_{r_{c}}$ bases, (b) $\delta \mathrm{S}_{Q}=\mathrm{S}_{Q}-\mathrm{S}_{Q}^{e q}$ where the $\mathrm{S}_{Q}^{e q}$ is taken at the equilibrium value of the lattice constant $a_{0}=10.2$ a.u.. $\diamond$ stands for $r_{c}=4$ a.u., + for $r_{c}=5$ a.u., $\square$ for $r_{c}=6$ a.u., $\times$ for $r_{c}=\infty$.

FIG. 13. Charge transfer in $\mathrm{SiC}$ as a function of the charge spilling of the $s p$ basis used to project the density. $\square$, stands for $\mathrm{PAO}_{\infty}$ optimized for the heteropolar system and independently for $\mathrm{Si}$ and $\mathrm{C}$ in a zinc-blende structure. $\diamond$, stands for STO3 basis in the same conditions, and + for a STO2 basis. 
TABLE I. Bases for silicon $(s p)$, diamond $(s p)$, and aluminum $(s p$ and $s p d) . \mathcal{S}_{Q}$ stands for the charge spilling, and $\mathcal{S}_{4}$ and $\mathcal{S}_{8}$ for the spilling considering the first 4 and 8 bands, respectively. $\mathrm{PAO}_{\infty}$ and $\mathrm{PAO}_{r_{c}}$ are fixed bases, whereas STO1, STO2, STO3, and $\mathrm{PAO}_{\infty}^{\text {opt }}$ have been optimized according to their respective variational parameters, to minimize $\mathcal{S}_{Q} \cdot Q_{s}$, $Q_{p}$, and $Q_{d}$ are the total charges assigned to $s, p$, and $d$ orbitals. The mean square error of the LCAO bands with respect to the $\mathrm{PW}$ reference bands (in $\mathrm{eV}$ ) is displayed for the valence bands $\left(\sigma_{v}\right)$ and two first conduction bands $\left(\sigma_{c}\right)$ for silicon and diamond, and for the first and second bands $\left(\sigma_{1+2}\right)$ and third and forth $\left(\sigma_{3+4}\right)$ for aluminum. Min $\mathcal{S}$ corresponds to the spilling lower bound (see text).

\begin{tabular}{|c|c|c|c|c|c|c|c|c|c|}
\hline & Basis & & $\mathcal{S}_{Q}$ & $\mathcal{S}_{8}$ & $Q_{s}$ & $Q_{p}$ & & $\sigma_{v}$ & $\sigma_{c}$ \\
\hline & $\mathrm{PAO}_{\infty}$ & $s p$ & 0.0080 & 0.1596 & 1.32 & 2.68 & & 0.30 & 2.15 \\
\hline & $\mathrm{PAO}_{\infty}^{o p t}$ & $s p$ & 0.0078 & 0.1621 & 1.35 & 2.65 & & 0.29 & 1.97 \\
\hline & $\mathrm{PAO}_{5.0}$ & $s p$ & 0.0139 & 0.1591 & 1.40 & 2.60 & & 0.36 & 2.09 \\
\hline \multirow[t]{9}{*}{$\mathrm{Si}$} & STO1 & $s p$ & 0.0109 & 0.1764 & 1.48 & 2.52 & & 0.38 & 2.43 \\
\hline & STO2 & $s p$ & 0.0076 & 0.1562 & 1.36 & 2.64 & & 0.34 & 2.34 \\
\hline & STO3 & $s p$ & 0.0074 & 0.1548 & 1.34 & 2.66 & & 0.29 & 1.95 \\
\hline & $\operatorname{Min} \mathcal{S}$ & $s p$ & 0.0044 & 0.0689 & & & & & \\
\hline & $\operatorname{Min} \mathcal{S}$ & spd & 0.0001 & 0.0014 & & & & & \\
\hline & Basis & & $\mathcal{S}_{Q}$ & $\mathcal{S}_{8}$ & $Q_{s}$ & $Q_{p}$ & & $\sigma_{v}$ & $\sigma_{c}$ \\
\hline & $\mathrm{PAO}_{\infty}$ & $s p$ & 0.0035 & 0.0719 & 1.05 & 2.95 & & 0.32 & 2.19 \\
\hline & $\mathrm{PAO}_{\infty}^{o p t}$ & $s p$ & 0.0027 & 0.0780 & 1.07 & 2.93 & & 0.25 & 2.61 \\
\hline & $\mathrm{PAO}_{4.0}$ & $s p$ & 0.0041 & 0.0672 & 1.10 & 2.90 & & 0.23 & 2.22 \\
\hline \multirow[t]{5}{*}{$\mathrm{C}$} & STO1 & $s p$ & 0.0039 & 0.0771 & 1.11 & 2.89 & & 0.26 & 3.07 \\
\hline & STO2 & $s p$ & 0.0039 & 0.0775 & 1.12 & 2.88 & & 0.24 & 3.05 \\
\hline & STO3 & $s p$ & 0.0024 & 0.0790 & 1.09 & 2.98 & & 0.17 & 3.36 \\
\hline & $\operatorname{Min} \mathcal{S}$ & $s p$ & 0.0008 & 0.0226 & & & & & \\
\hline & Basis & & $\mathcal{S}_{Q}$ & $\mathcal{S}_{4}$ & $Q_{s}$ & $Q_{p}$ & $Q_{d}$ & $\sigma_{1+2}$ & $\sigma_{3+4}$ \\
\hline & $\mathrm{PAO}_{\infty}$ & $s p$ & 0.0165 & 0.1141 & 1.13 & 2.87 & & 2.03 & 3.45 \\
\hline & $\mathrm{PAO}_{\infty}$ & spd & 0.0022 & 0.0053 & 0.77 & 1.39 & 0.84 & 0.61 & 0.24 \\
\hline & $\mathrm{PAO}_{\infty}^{o p t}$ & spd & 0.0011 & 0.0079 & 0.98 & 1.41 & 0.61 & 0.35 & 0.84 \\
\hline & $\mathrm{PAO}_{6.0}$ & $s p$ & 0.0157 & 0.1012 & 1.30 & 1.70 & & 1.54 & 2.97 \\
\hline \multirow[t]{5}{*}{$\mathrm{Al}$} & $\mathrm{PAO}_{6.0}$ & spd & 0.0014 & 0.0042 & 1.02 & 1.39 & 0.59 & 0.20 & 0.45 \\
\hline & $\mathrm{PAO}_{5.3}$ & $s p$ & 0.0158 & 0.0930 & 1.34 & 1.66 & & 1.34 & 2.63 \\
\hline & $\mathrm{PAO}_{5.3}$ & spd & 0.0007 & 0.0020 & 1.15 & 1.45 & 0.40 & 0.97 & 2.15 \\
\hline & $\operatorname{Min} \mathcal{S}$ & $s p$ & 0.0009 & 0.0025 & & & & & \\
\hline & $\operatorname{Min} \mathcal{S}$ & spd & $2.910^{-5}$ & 0.0007 & & & & & \\
\hline
\end{tabular}

TABLE II. Basis optimizations for different number of bands, for $\mathrm{Si}, \mathrm{C}$ (diamond), BN, and Al. Superindex indicates the number of bands used for the $\mathcal{S}$ minimization $(v$ means the valence bands). The basis is minimal, except for $\mathrm{Al}$, for which it is spd. $\mathcal{S}_{Q}$ is the charge spilling, and $\mathcal{S}_{4}$ and $\mathcal{S}_{8}$ are the spilling considering the first 4 and 8 bands, respectively. The mean square error of the LCAO bands with respect to the $\mathrm{PW}$ reference bands (in $\mathrm{eV}$ ) is displayed for the valence bands $\left(\sigma_{v}\right)$ and two first conduction bands $\left(\sigma_{c}\right)$ for $\mathrm{Si}, \mathrm{C}$, and $\mathrm{BN}$, and for the first and second bands $\left(\sigma_{1+2}\right)$ and third and forth $\left(\sigma_{3+4}\right)$ for Al. $E_{g a p}^{\Gamma}$ and $E_{g a p}$ are the direct gap at $\Gamma$ and the absolute gap, respectively, both in $\mathrm{eV}$. $\mathbf{k}$ is the wave vector of the minimum of the conduction band in units of $\pi / a$, being $|\mathbf{k}|=1$ for the $\mathrm{X}$ point.

\begin{tabular}{|c|c|c|c|c|c|c|c|c|}
\hline & Basis & $\mathcal{S}_{Q}$ & $\mathcal{S}_{8}$ & $\sigma_{v}$ & $\sigma_{c}$ & $E_{g a p}^{\Gamma}$ & $E_{g a p}$ & $|\mathbf{k}|$ \\
\hline \multirow{12}{*}{$\mathrm{Si}$} & $\mathrm{PAO}_{\infty}^{v}$ & 0.0078 & 0.1621 & 0.28 & 1.97 & 2.91 & 1.69 & 0.63 \\
\hline & $\mathrm{PAO}_{\infty}^{8}$ & 0.0348 & 0.1233 & 0.94 & 2.13 & 2.33 & 1.44 & 0.80 \\
\hline & $\mathrm{PAO}_{5.0}^{v}$ & 0.0136 & 0.1453 & 0.44 & 2.16 & 2.78 & 1.96 & 0.62 \\
\hline & $\mathrm{PAO}_{5.0}^{8}$ & 0.0230 & 0.1170 & 0.91 & 2.47 & 2.50 & 0.95 & 0.83 \\
\hline & $\mathrm{STO}^{v}$ & 0.0109 & 0.1764 & 0.38 & 2.43 & 2.83 & 2.60 & 0.47 \\
\hline & $\mathrm{STO}^{8}$ & 0.0207 & 0.1541 & 0.60 & 2.27 & 2.40 & 1.94 & 0.60 \\
\hline & $\mathrm{STO} 2^{v}$ & 0.0076 & 0.1562 & 0.34 & 2.34 & 2.66 & 2.09 & 0.53 \\
\hline & $\mathrm{STO} 2^{8}$ & 0.0306 & 0.1179 & 0.79 & 1.48 & 2.39 & 0.85 & 0.80 \\
\hline & $\mathrm{STO}^{v}$ & 0.0074 & 0.1548 & 0.29 & 1.95 & 2.66 & 1.74 & 0.60 \\
\hline & STO $^{8}{ }^{8}$ & 0.0465 & 0.1121 & 1.20 & 1.28 & 2.52 & 0.53 & 0.83 \\
\hline & PW & & & & & 2.55 & 0.45 & 0.85 \\
\hline & Basis & $\mathcal{S}_{Q}$ & $\mathcal{S}_{8}$ & $\sigma_{v}$ & $\sigma_{c}$ & $E_{g a p}^{\Gamma}$ & $E_{\text {gap }}$ & $|\mathbf{k}|$ \\
\hline \multirow{10}{*}{$\mathrm{C}$} & $\mathrm{PAO}_{\infty}^{v}$ & 0.0027 & 0.0780 & 0.25 & 2.61 & 6.57 & 5.83 & 0.50 \\
\hline & $\mathrm{PAO}_{\infty}^{8}$ & 0.0192 & 0.0600 & 0.89 & 2.04 & 5.19 & 4.13 & 0.67 \\
\hline & $\mathrm{STO}^{v}$ & 0.0039 & 0.0771 & 0.26 & 3.07 & 5.78 & 5.78 & 0.00 \\
\hline & $\mathrm{STO}^{8}{ }^{8}$ & 0.0208 & 0.0592 & 0.94 & 1.95 & 4.67 & 4.34 & 0.42 \\
\hline & $\mathrm{STO}^{v}$ & 0.0039 & 0.0775 & 0.24 & 3.05 & 5.79 & 5.79 & 0.00 \\
\hline & STO $2^{8}$ & 0.0293 & 0.0545 & 1.37 & 1.88 & 4.49 & 3.51 & 0.58 \\
\hline & $\mathrm{STO}^{v}$ & 0.0024 & 0.0790 & 0.17 & 3.36 & 6.32 & 6.18 & 0.42 \\
\hline & $\mathrm{STO}^{8}$ & 0.0335 & 0.0526 & 1.32 & 1.46 & 4.81 & 3.96 & 0.67 \\
\hline & PW & & & & & 5.28 & 3.43 & 0.76 \\
\hline & Basis & $\mathcal{S}_{Q}$ & $\mathcal{S}_{8}$ & $\sigma_{v}$ & $\sigma_{c}$ & $E_{g a p}^{\Gamma}$ & $E_{g a p}$ & $|\mathbf{k}|$ \\
\hline \multirow{4}{*}{$\mathrm{BN}$} & $\mathrm{PAO}_{\infty}^{v}$ & 0.0022 & 0.0766 & 0.24 & 3.06 & 10.90 & 8.56 & 0.93 \\
\hline & $\mathrm{PAO}_{\infty}^{8}$ & 0.0154 & 0.0534 & 0.96 & 2.21 & 7.93 & 4.63 & 0.93 \\
\hline & PW & & & & & 8.19 & 3.82 & 0.93 \\
\hline & Basis & $\mathcal{S}_{Q}$ & $\mathcal{S}_{4}$ & $\sigma_{1+2}$ & $\sigma_{3+4}$ & & & \\
\hline \multirow[t]{2}{*}{$\mathrm{Al}$} & $\mathrm{PAO}_{\infty}^{v}$ & 0.0011 & 0.0079 & 0.35 & 0.84 & & & \\
\hline & $\mathrm{PAO}_{\infty}^{4}$ & 0.0036 & 0.0024 & 0.37 & 0.23 & & & \\
\hline
\end{tabular}


TABLE III. Interaction range for minimal $s p$ bases in Si. NS stands for the number of neighbor shells with non-negligible interactions and $r_{99.9 \%}$ is a measure of the size of the orbitals as defined in the text. $\mathcal{S}_{Q}$ stands for the charge spilling, and $\mathcal{S}_{8}$ for the spilling considering the first 8 bands. The mean square error of the LCAO bands with respect to the PW reference bands (in $\mathrm{eV}$ ) is displayed for the valence bands $\left(\sigma_{v}\right)$ and two first conduction bands $\left(\sigma_{c}\right)$. STO1 1.75 and $\mathrm{STO}_{1.85}$ are the STO1 bases for the exponents $\beta=1.75$ a.u. ${ }^{-1}$ (Ref. 5) and 1.85, respectively.

\begin{tabular}{lllllll}
\hline \hline Basis & $\mathcal{S}_{Q}$ & $\mathcal{S}_{8}$ & $\sigma_{v}$ & $\sigma_{c}$ & $r_{99.9 \%}$ & $\mathrm{NS}$ \\
\hline STO1 $_{1.75}$ & 0.0319 & 0.2279 & 0.96 & 4.27 & 5.1 & 4 \\
STO1 $_{1.85}$ & 0.0514 & 0.2533 & 1.07 & 4.64 & 4.8 & 3 \\
& & & & & & \\
PAO $_{\infty}$ & 0.0080 & 0.1596 & 0.30 & 2.15 & 7.9 & 9 \\
PAO $_{5.0}$ & 0.0139 & 0.1591 & 0.36 & 2.09 & 4.6 & 3 \\
PAO $_{4.0}$ & 0.0506 & 0.2269 & 1.01 & 3.89 & 3.7 & 2 \\
\hline \hline
\end{tabular}

TABLE IV. Single- $z$, double- $z(2-z)$, and $s p d$ for Si, C (diamond). $\mathcal{S}_{Q}$ stands for the charge spilling, and $\mathcal{S}_{8}$ for the spilling considering the first 8 bands. The mean square error of the LCAO bands with respect to the PW reference bands (in $\mathrm{eV}$ ) is given for the valence bands $\left(\sigma_{v}\right)$ and two first conduction bands $\left(\sigma_{c}\right)$. Superscript $v$ means optimized for the valence. STO 2- $z$ bases after Ref. 21.

\begin{tabular}{lllllll}
\hline \hline & Basis & & $\mathcal{S}_{Q}$ & $\mathcal{S}_{8(4)}$ & $\sigma_{v}$ & $\sigma_{c}$ \\
\hline & & & & & & \\
& STO3 $^{v}$ & $s p$ & 0.0074 & 0.1548 & 0.29 & 1.95 \\
& STO & $2-z$ & 0.0099 & 0.0735 & 0.36 & 1.50 \\
& STO3 $^{v}$ & $s p d$ & 0.0009 & 0.0101 & 0.09 & 0.23 \\
& & & & & & \\
& PAO $_{5.0}$ & $s p$ & 0.0139 & 0.1591 & 0.36 & 2.09 \\
& PAO $_{5.0}$ & $2-z$ & 0.0085 & 0.0827 & 0.36 & 1.50 \\
& PAO $_{5.0}$ & $s p d$ & 0.0042 & 0.0132 & 0.15 & 0.38 \\
\hline & & & & & & \\
& STO3 $^{v}$ & $s p$ & 0.0024 & 0.0790 & 0.17 & 3.36 \\
& STO $_{\mathrm{C}}$ & $2-z$ & 0.0032 & 0.0059 & 0.36 & 0.40 \\
& & & & & & \\
& PAO $_{4.0}$ & $s p$ & 0.0041 & 0.0672 & 0.23 & 2.22 \\
& PAO $_{4.0}$ & $2-z$ & 0.0018 & 0.0165 & 0.28 & 1.37 \\
\hline \hline
\end{tabular}

TABLE V. Band gap in $\mathrm{Si}$ and $\mathrm{C}$ (diamond) for single- $z$ and double- $z(2-z)$ bases. $E_{g a p}^{\Gamma}$ and $E_{g a p}$ stand for the direct gap energy at $\Gamma$ and for the absolute gap energy, respectively, both in $\mathrm{eV} . \mathbf{k}$ is the wave vector of the minimum of the conduction band in units of $\pi / a$, being $|\mathbf{k}|=1$ for the $\mathrm{X}$ point.

\begin{tabular}{|c|c|c|c|c|c|}
\hline & Basis & & $E_{g a p}^{\Gamma}$ & $E_{\text {gap }}$ & $|\mathbf{k}|$ \\
\hline \multirow{7}{*}{$\mathrm{Si}$} & $\mathrm{STO}^{v}$ & $s p$ & 2.65 & 1.74 & 0.60 \\
\hline & STO & $2-z$ & 2.40 & 0.88 & 0.73 \\
\hline & $\mathrm{STO}^{v}$ & spd & 2.58 & 0.76 & 0.85 \\
\hline & $\mathrm{PAO}_{5.0}$ & $s p$ & 2.47 & 2.18 & 0.47 \\
\hline & $\mathrm{PAO}_{5.0}$ & $2-z$ & 2.49 & 1.03 & 0.70 \\
\hline & $\mathrm{PAO}_{5.0}$ & spd & 2.65 & 0.91 & 0.85 \\
\hline & PW & & 2.55 & 0.45 & 0.85 \\
\hline \multirow{5}{*}{$\mathrm{C}$} & $\mathrm{STO}^{v}$ & $s p$ & 6.32 & 6.18 & $\overline{0.41}$ \\
\hline & STO & $2-z$ & 5.46 & 4.30 & 0.73 \\
\hline & $\mathrm{PAO}_{4.0}$ & $s p$ & 5.98 & 5.89 & 0.34 \\
\hline & $\mathrm{PAO}_{4.0}$ & $2-z$ & 6.00 & 5.28 & 0.53 \\
\hline & PW & & 5.28 & 3.43 & 0.76 \\
\hline
\end{tabular}

TABLE VI. Calculated charge transfer using optimized $\mathrm{PAO}_{\infty}^{\text {opt }}$ basis for some zincblende semiconductors. $Q_{C}$ and $Q_{A}$ stand for the valence charge on the cation and the anion, respectively, $\delta Q$ for the charge transfer with respect to neutral atoms, and $\mathcal{S}_{Q}$ for the charge spilling. Numbers in parenthesis were obtained from Hartree-Fock LCAO calculations, ${ }^{22}$ except for GaAs, for which LDA LCAO was used. ${ }^{23}$

\begin{tabular}{llllll}
\hline \hline & Basis & $\mathcal{S}_{Q}$ & $Q_{C}$ & $Q_{A}$ & $\delta Q$ \\
\hline $\mathrm{BN}$ & $s p$ & 0.0022 & $2.19(2.14)$ & $5.81(5.86)$ & $0.81(0.86)$ \\
$\mathrm{BP}$ & $s p$ & 0.0038 & $3.51(3.34)$ & $4.49(4.66)$ & $-.51(-.34)$ \\
$\mathrm{AlP}$ & $s p$ & 0.0035 & $2.15(2.20)$ & $5.85(5.80)$ & $0.85(0.80)$ \\
$\mathrm{SiC}$ & $s p$ & 0.0071 & $2.30(2.19)$ & $5.70(5.81)$ & $1.70(1.81)$ \\
$\mathrm{GaAs}$ & $s p$ & 0.0041 & 2.58 & 5.42 & 0.42 \\
& $s p d$ & 0.0010 & $2.78(2.88)$ & $5.22(5.12)$ & $0.22(0.12)$ \\
\hline \hline
\end{tabular}

TABLE VII. Calculated excess charge, in units of $e$, for the upper and lower $\mathrm{Si}$ atom in the dimer of a $\mathrm{Si}(100)-2 \times 1$ surface. A minimal $s p$ basis has been used in all cases. $\mathcal{S}_{Q}$ stands for the charge spilling. $\mathrm{PAO}_{\infty}^{\text {opt }}$ and STO1 have been optimized for bulk silicon. STO1 $1_{1.75}$ is a STO1 basis with $\beta=1.75$ a.u. ${ }^{-1}$ (Ref. 5).

\begin{tabular}{llll}
\hline \hline Basis & $\mathcal{S}_{Q}$ & $\delta Q_{\text {up }}$ & $\delta Q_{\text {down }}$ \\
\hline PAO $_{\infty}$ & 0.0080 & -0.10 & +0.12 \\
PAO $_{\infty}^{o p t}$ & 0.0078 & -0.10 & +0.13 \\
PAO $_{5.0}$ & 0.0139 & -0.13 & +0.10 \\
& & & \\
STO1 $_{1.75}$ & 0.0318 & -0.15 & +0.10 \\
STO1 & 0.0076 & -0.13 & +0.12 \\
\hline \hline
\end{tabular}



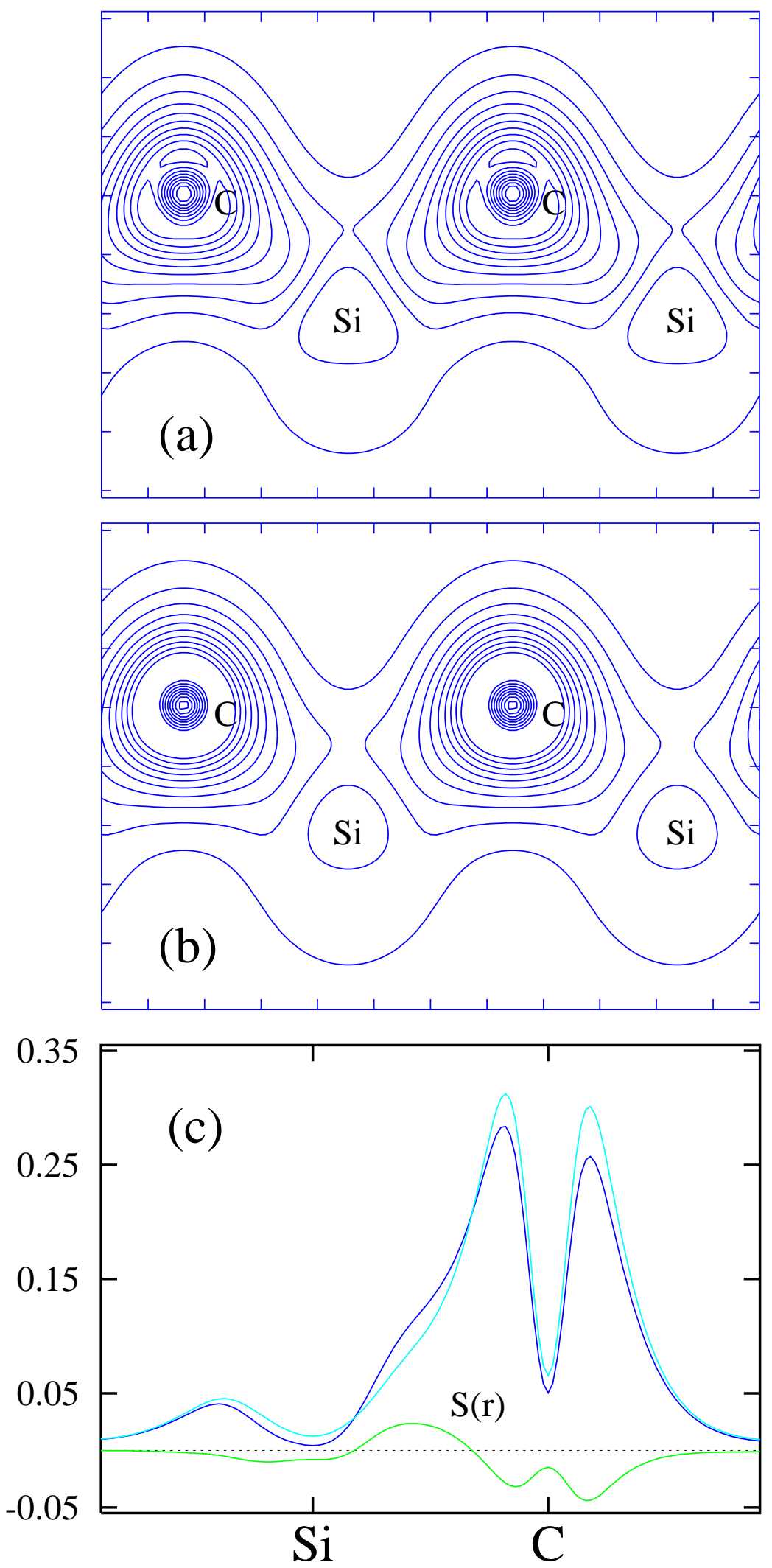


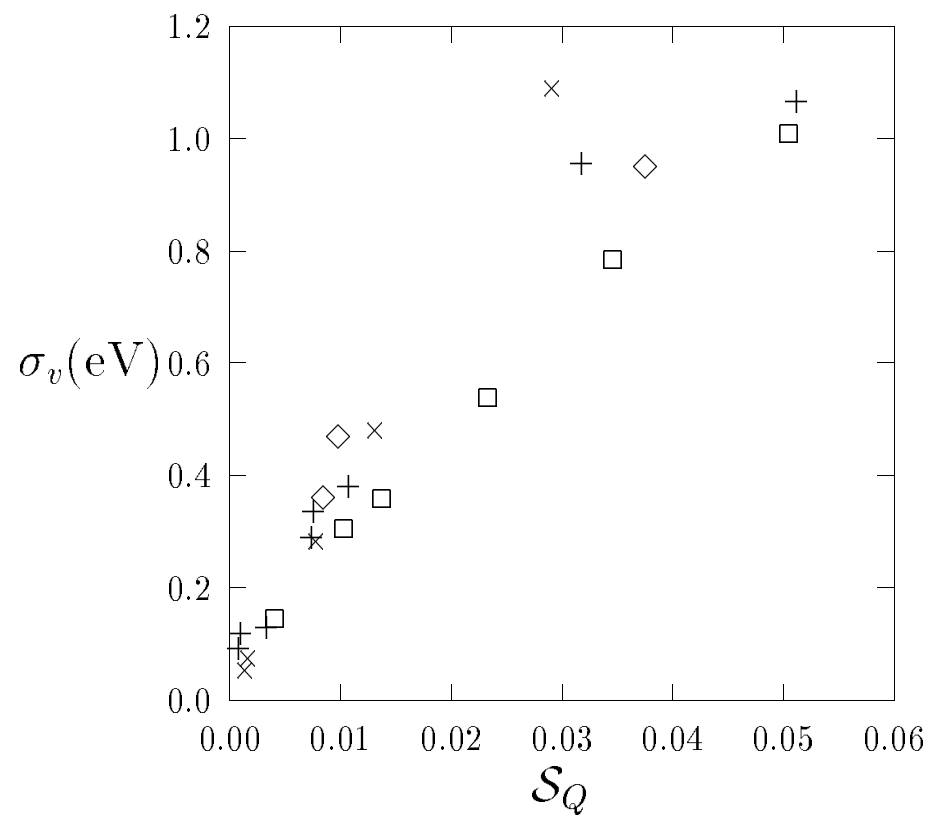



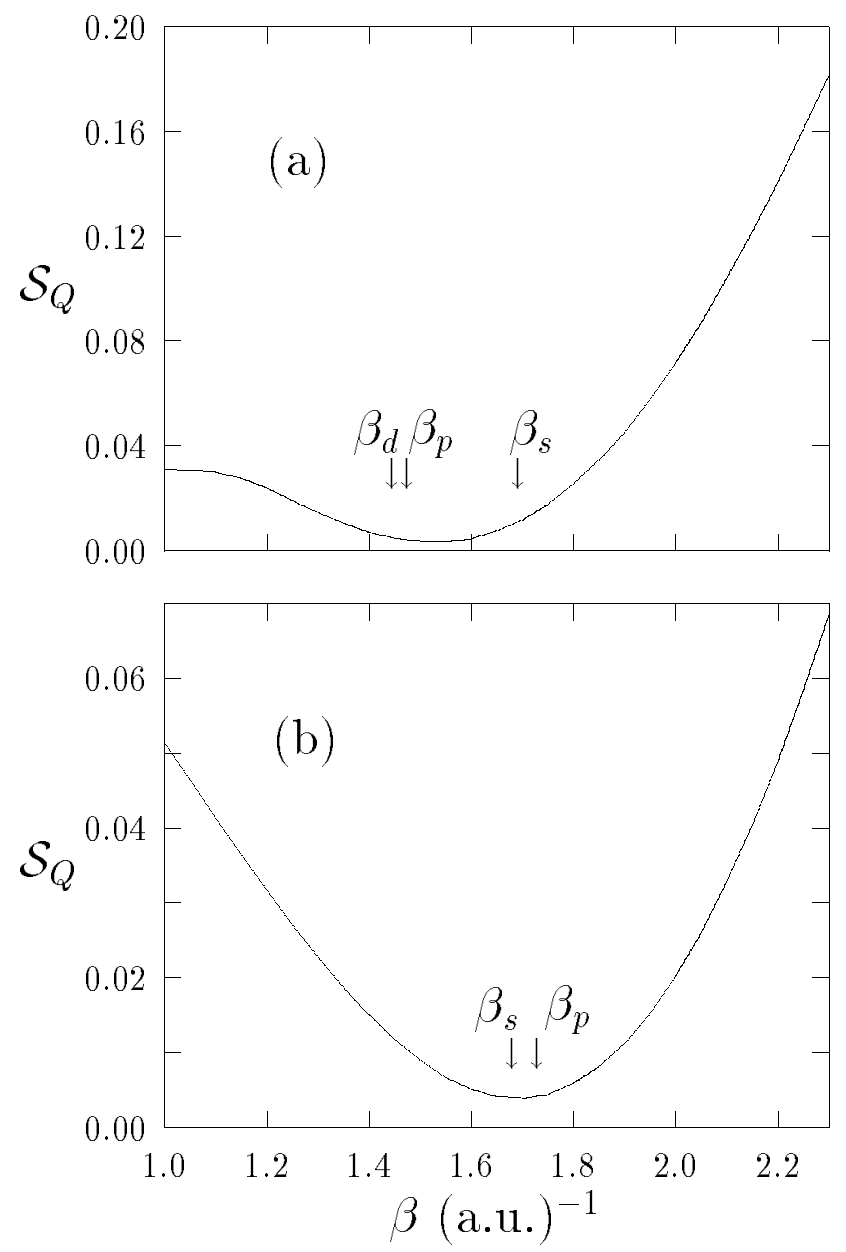


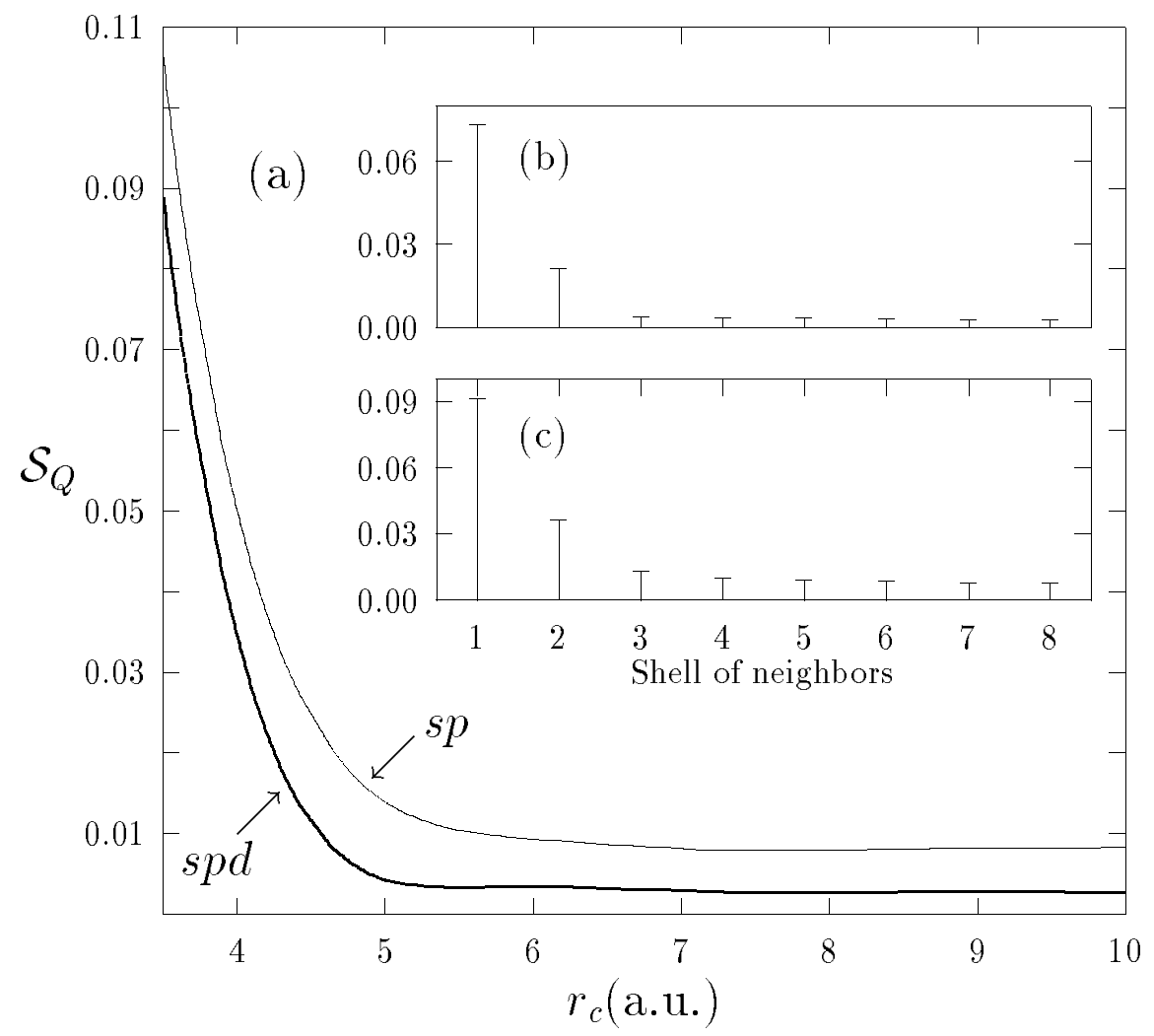




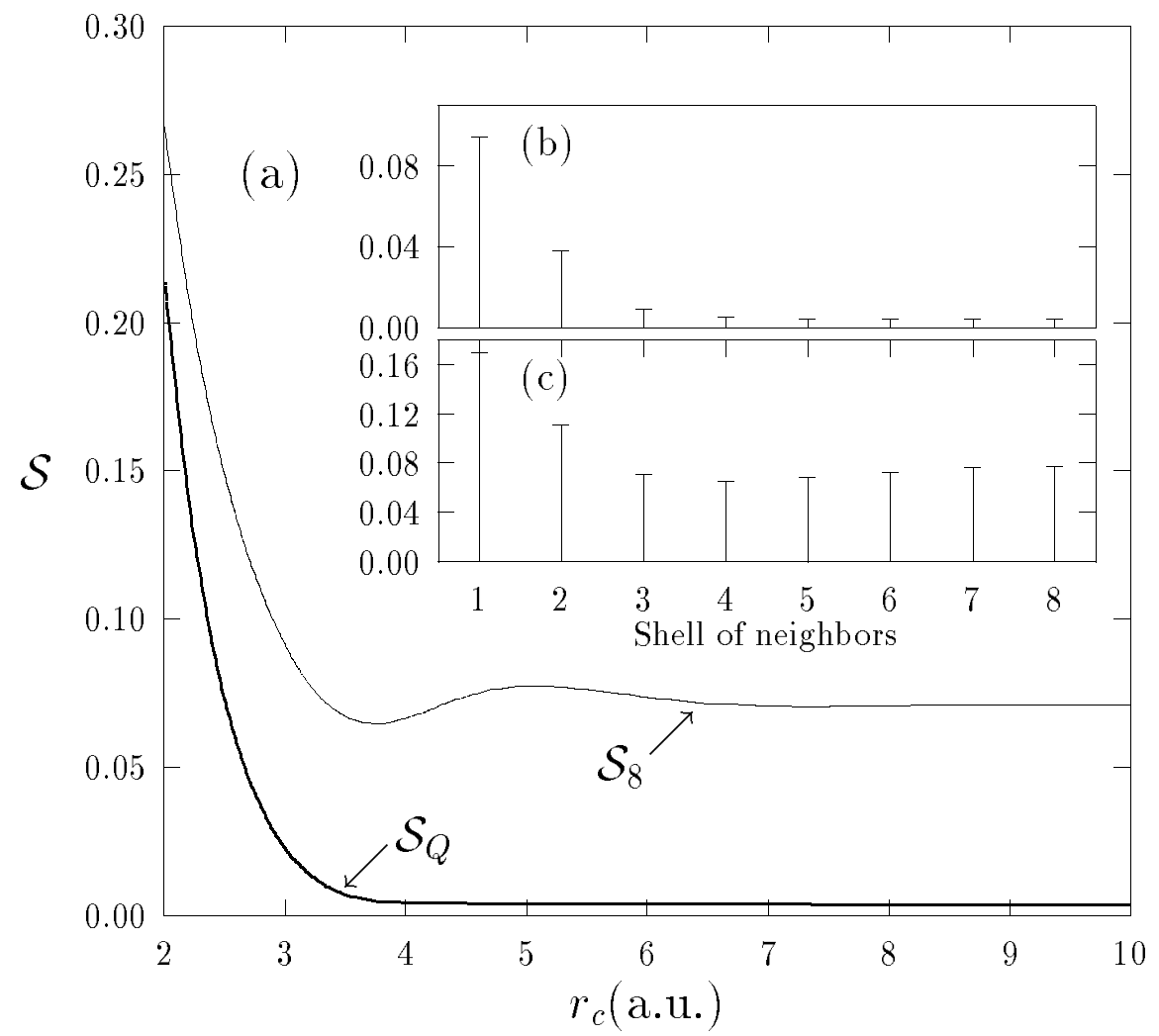




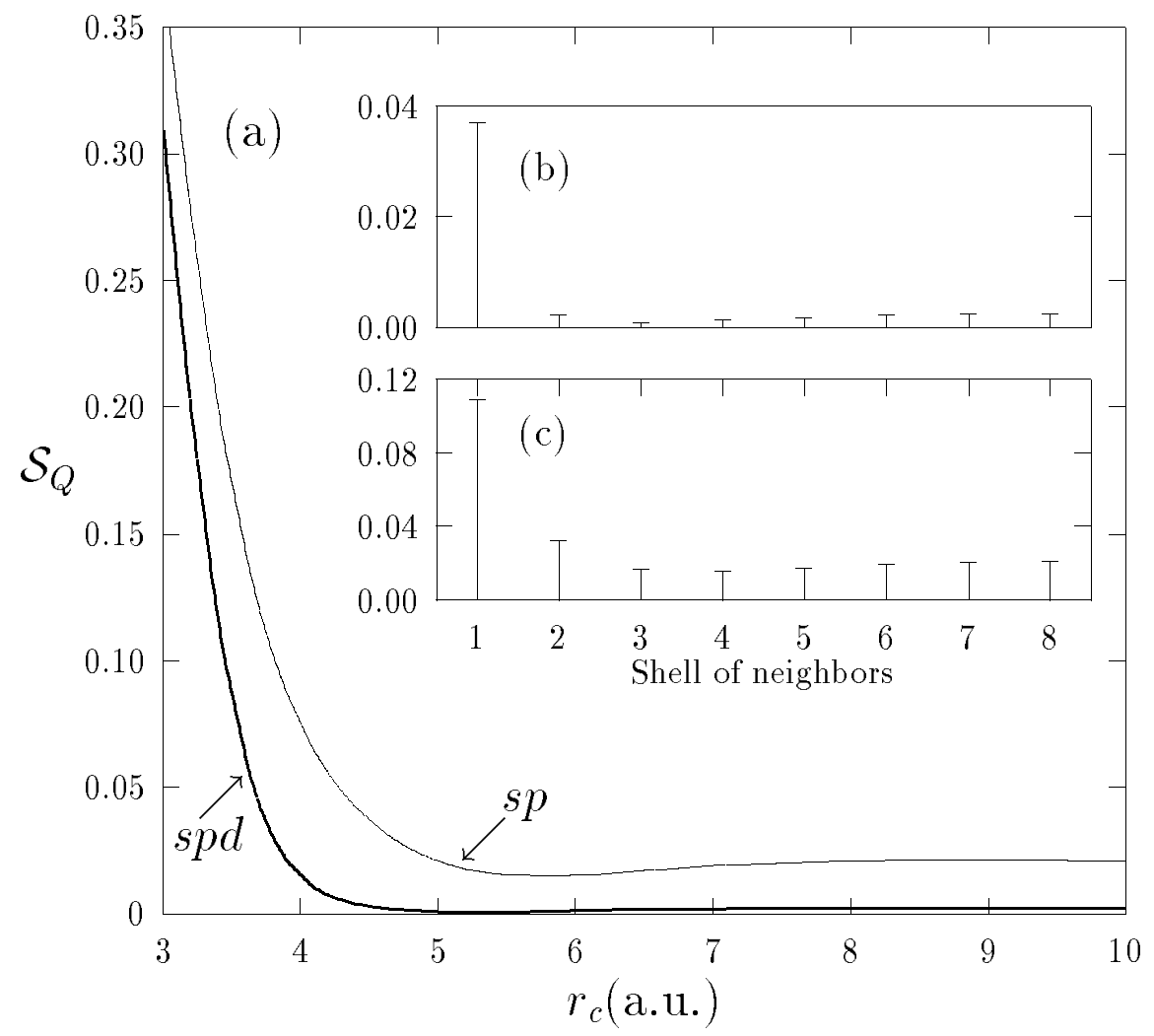




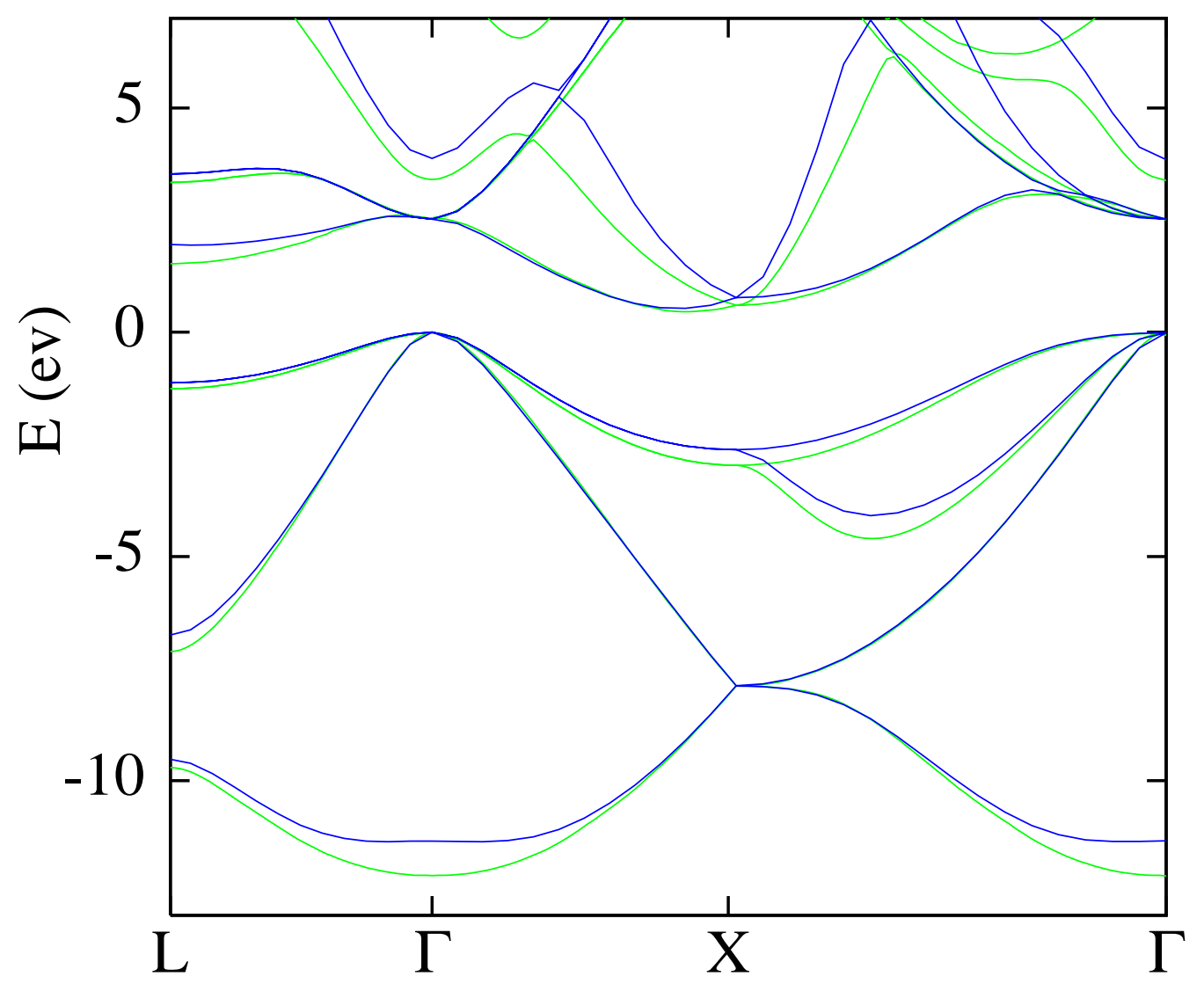



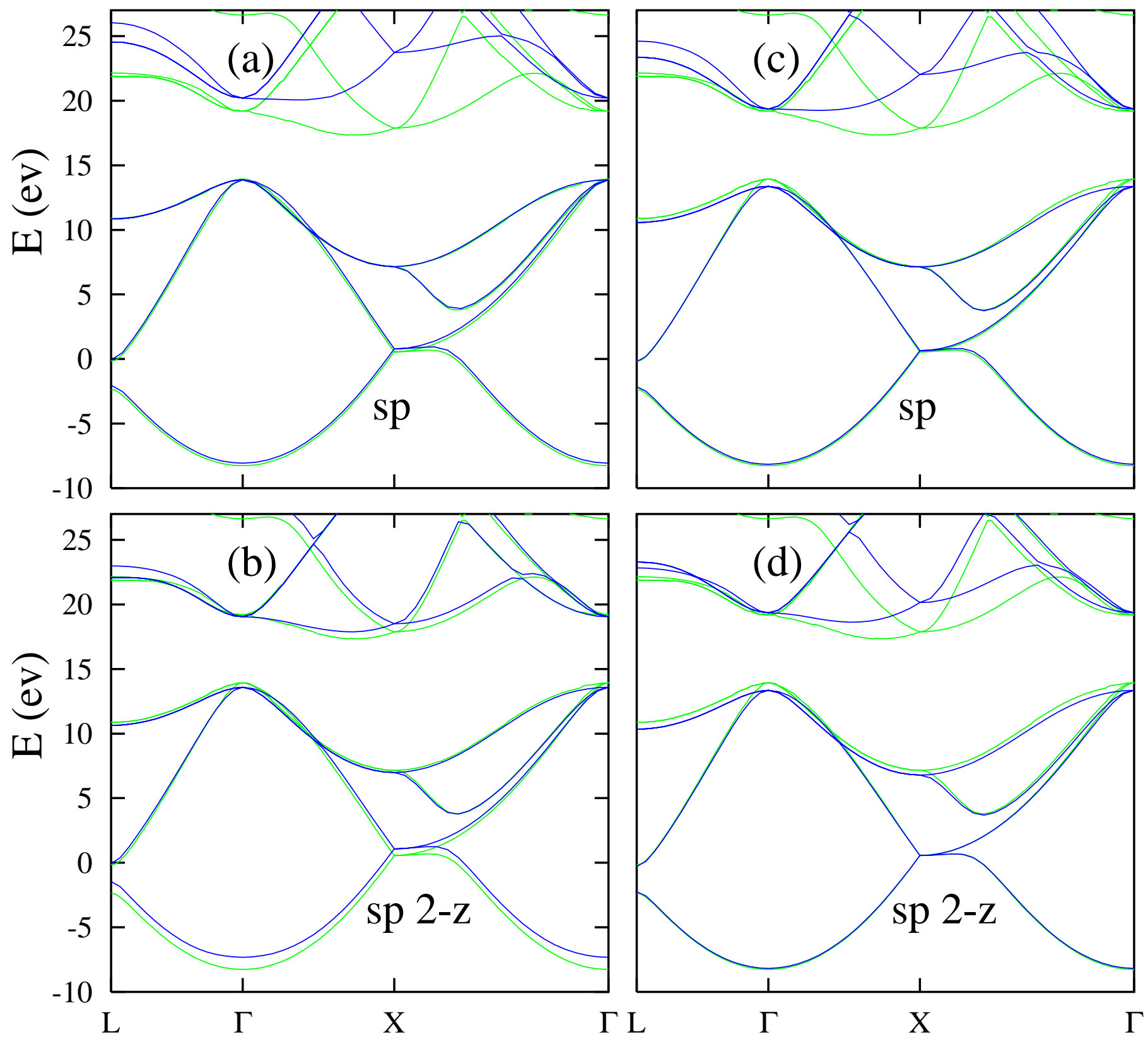


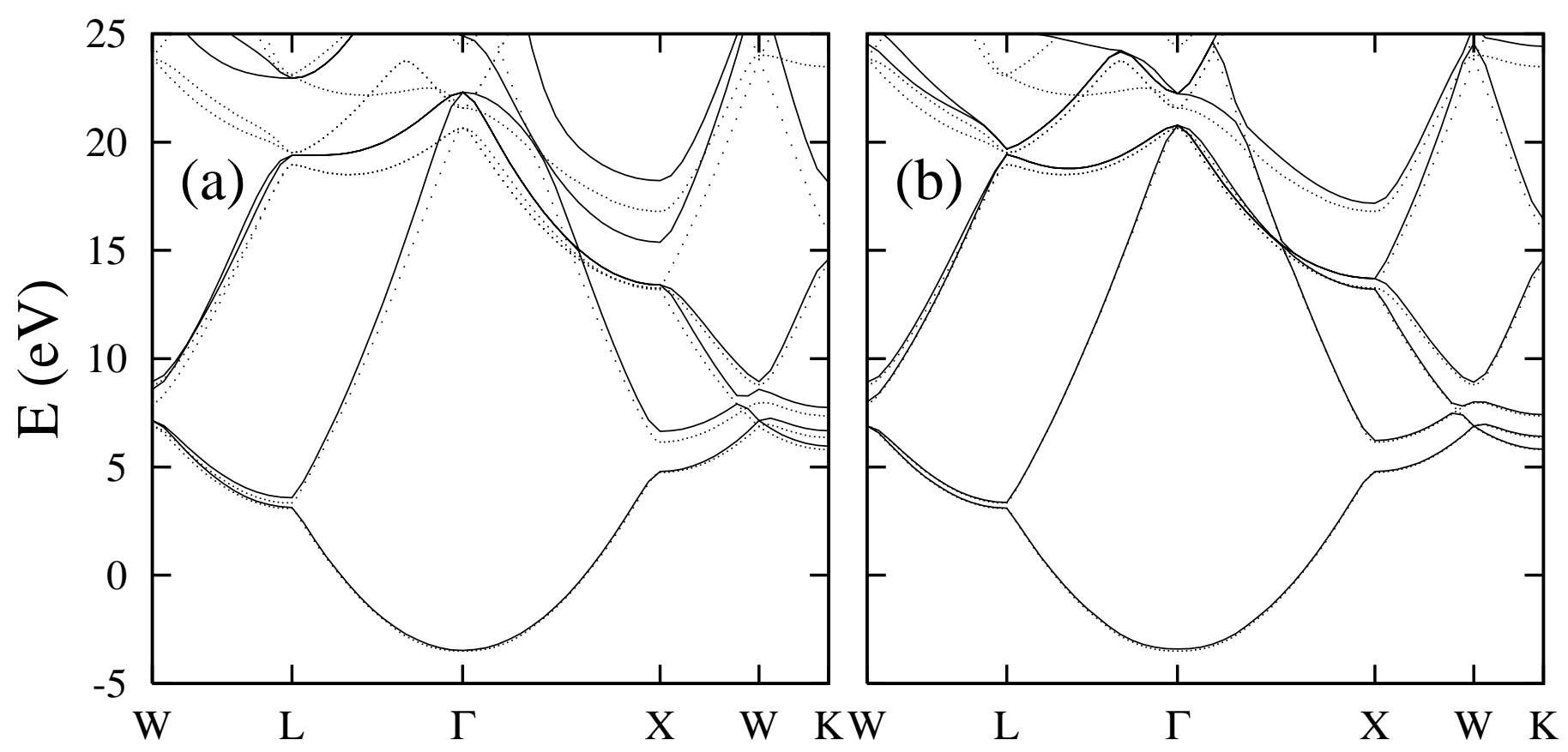




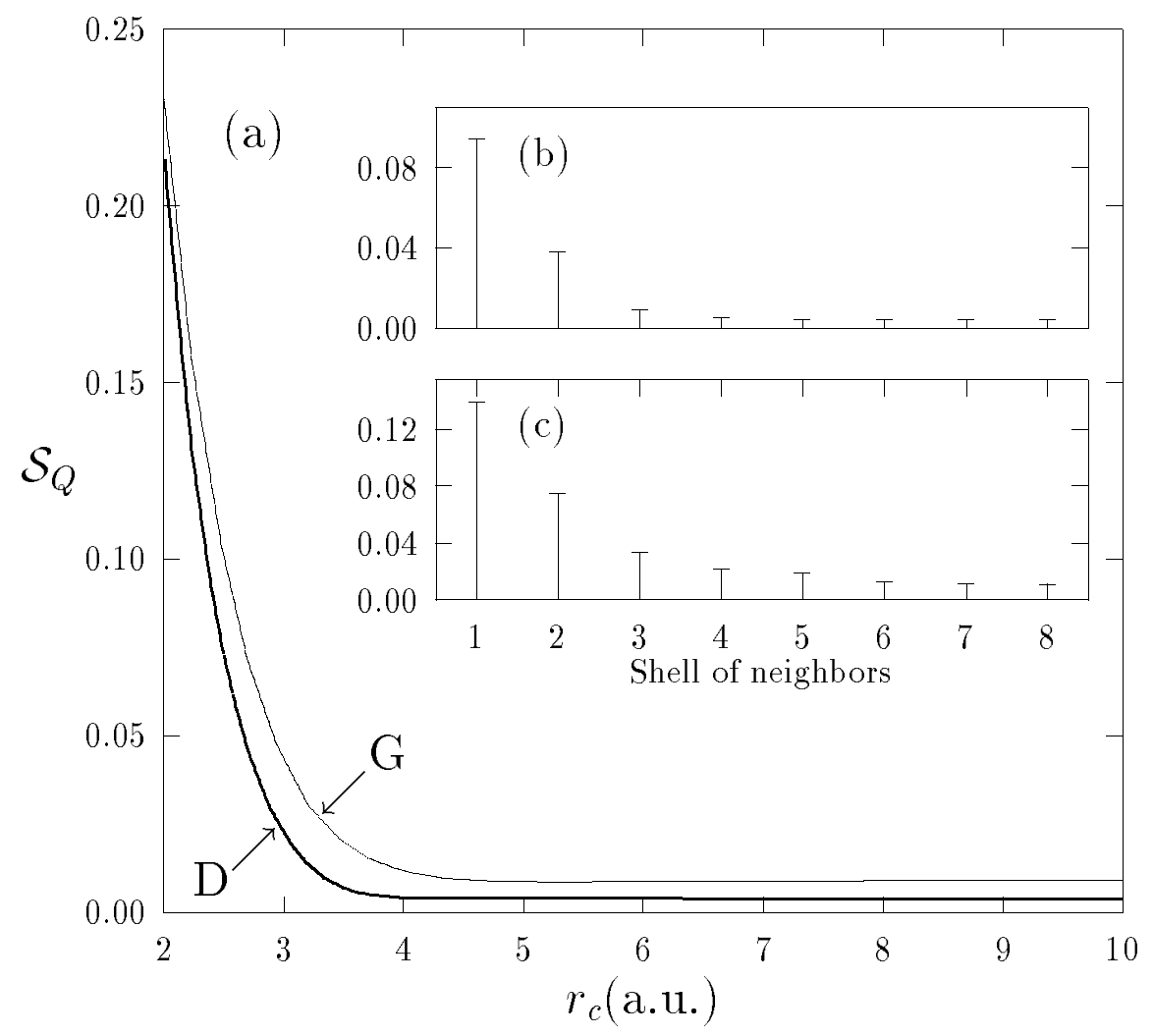



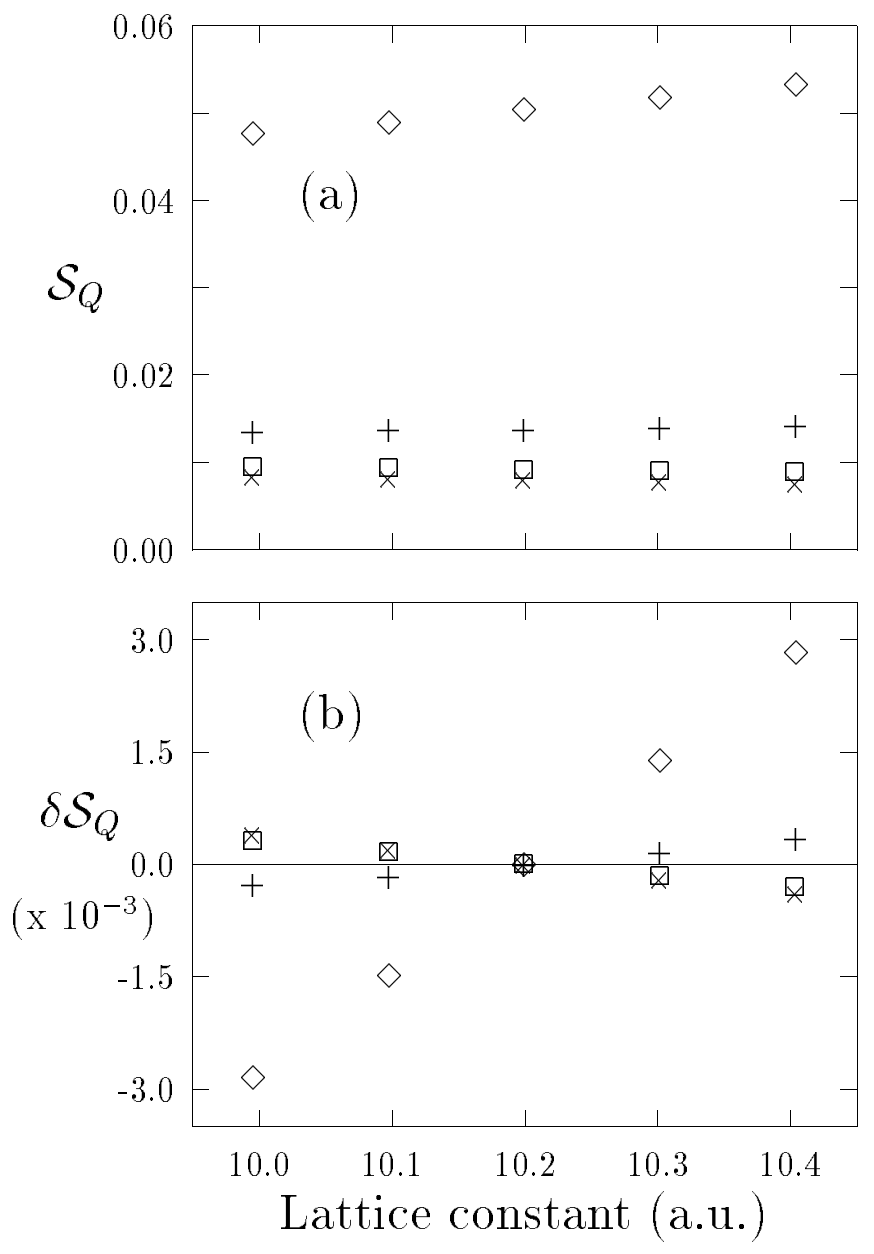


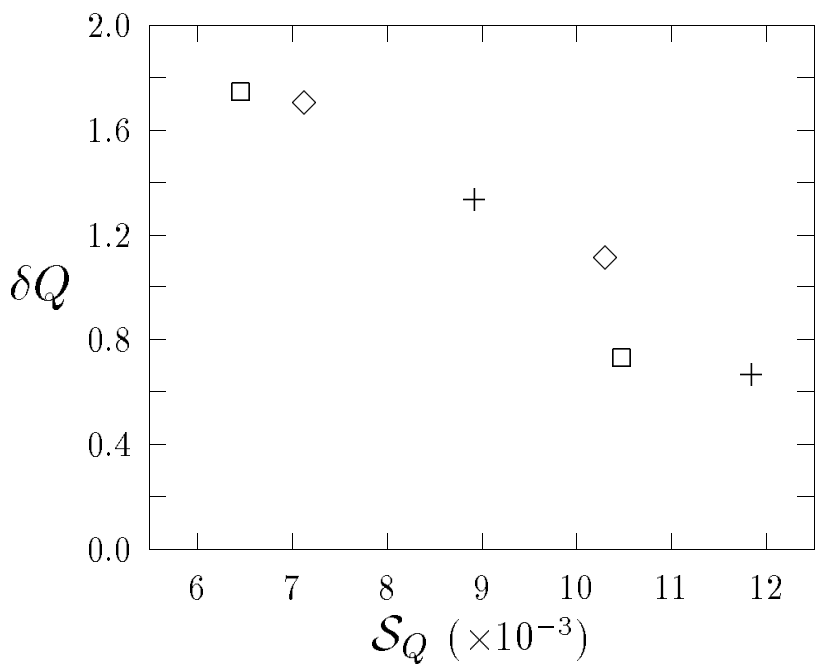

